\title{
Design And Expected Performance Of The AGR-1 Fission Product Monitoring System
}

\author{
J. K. Hartwell \\ D. M. Scates \\ M. W. Drigert
}

September 2005

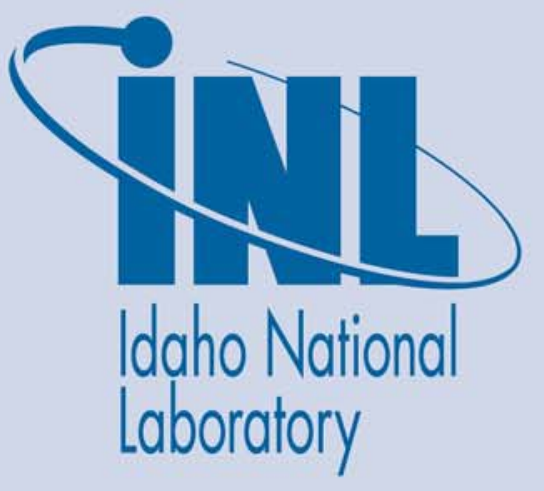

The INL is a U.S. Department of Energy National Laboratory operated by Battelle Energy Alliance 


\title{
Design and Expected Performance of the AGR-1 Fission Product Monitoring System
}

\author{
J. K. Hartwell \\ D. M. Scates \\ M. W. Drigert
}

September 2005

Idaho National Laboratory

Idaho Falls, Idaho 83415

Prepared for the

U.S. Department of Energy

Office of Nuclear Energy Science and Technology

NE-20, Advanced Nuclear Research

Under DOE Idaho Operations Office

Contract DE-AC07-05ID14517 


\section{ABSTRACT}

The US Department of Energy (DOE) is embarking on a series of tests of coated-particle reactor fuel for the Advanced Gas Reactor (AGR). As one part of this fuel development program, a series of eight (8) fuel irradiation tests are planned for the Idaho National Laboratory's (INL's) Advanced Test Reactor (ATR). The first test in this series (AGR-1) will incorporate six separate capsules irradiated simultaneously, each containing about 51,000 TRISO-coated fuel particles supported in a graphite matrix and continuously swept with inert gas during irradiation. The effluent gas from each of the six capsules must be independently monitored in near real time and the activity of various fission gas nuclides determined and reported.

A set of seven heavily-shielded, high-purity germanium (HPGe) gammaray spectrometers and sodium iodide $[\mathrm{NaI}(\mathrm{Tl})]$ scintillation detector-based total radiation detectors have been designed, and are being configured and tested for use during the AGR-1 experiment. The AGR-1 test specification requires that the fission product measurement system (FPMS) have sufficient sensitivity to detect the failure of a single coated fuel particle and sufficient range to allow it to "count" multiple (up to 250) successive particle failures. This paper describes the design AGR-1 FPMS, and details the calculations carried out to evaluate the expected performance of the system. 


\section{CONTENTS}

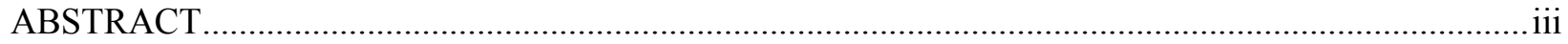

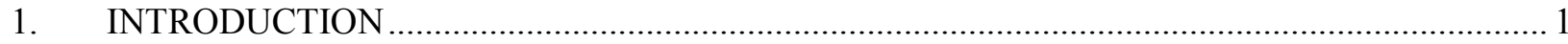

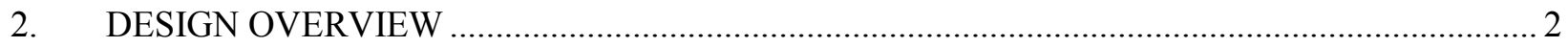

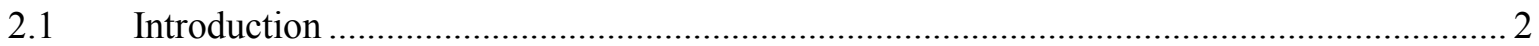

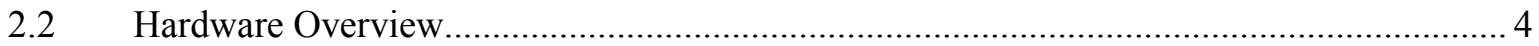

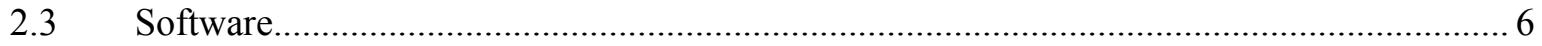

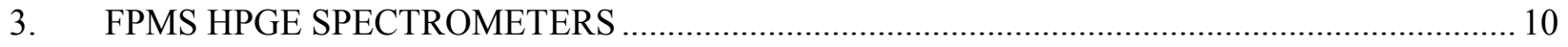

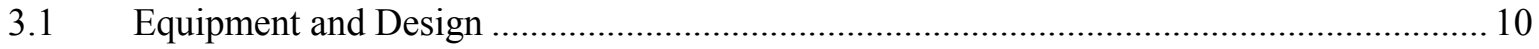

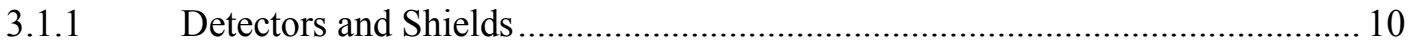

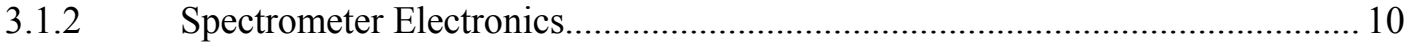

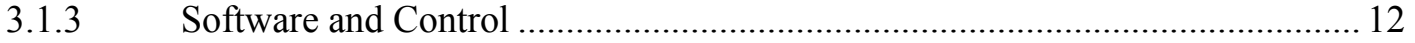

3.2 Expected Releases and Detection Sensitivity ............................................................. 12

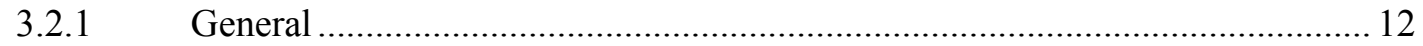

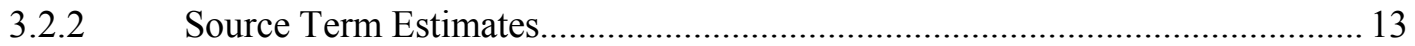

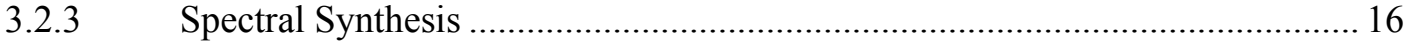

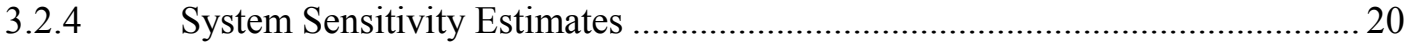

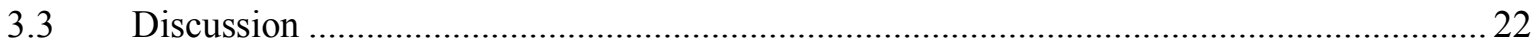

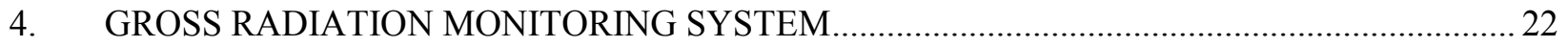

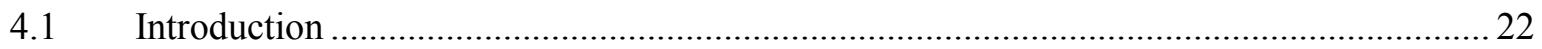

4.2 $\mathrm{NaI}(\mathrm{Tl})$ Gross Detection System Design ................................................................. 23

4.3 Gross Monitor Sensitivity Calculations..................................................................... 26

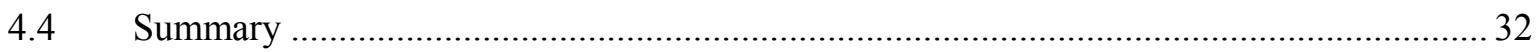

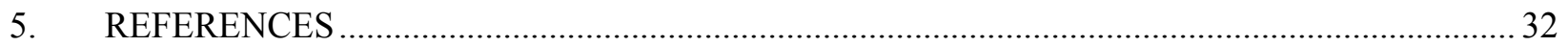

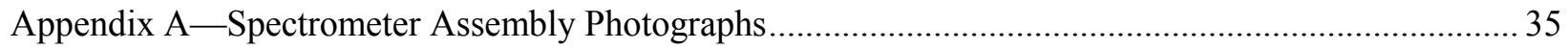




\section{FIGURES}

1. Overview depiction of the sampling system flow path ........................................................ 2

2. A sketch of the FPMS shield and detector arrangement............................................................ 3

3. Six gamma-ray spectrometers presently installed in the ATR ….............................................. 4

4. Hardware components of the Fission Product Monitor System (FPMS) and the flow of control and data communications between the different component sub-systems ............................ 5

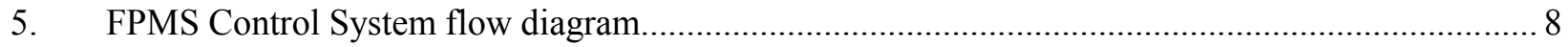

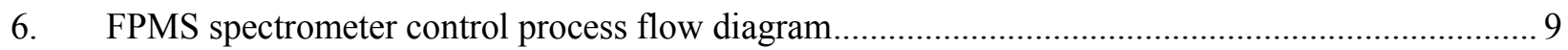

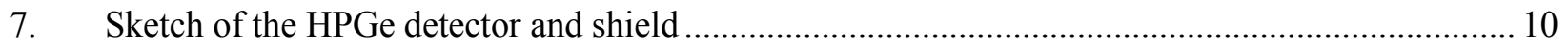

8. Partial cutaway sketch of an FPMS station showing the relative positioning of certain

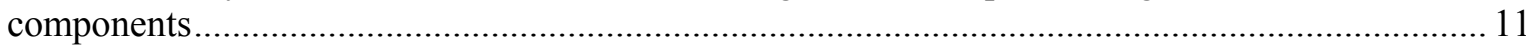

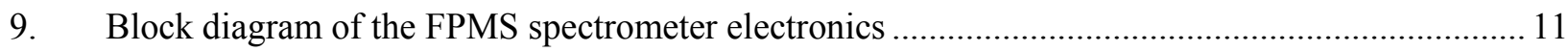

10. Inventory data for ${ }^{85 \mathrm{~m}} \mathrm{Kr}$ presenting the calculated MCWO results and the fitted values used in this work

11. A comparison of the ${ }^{85 \mathrm{~m}} \mathrm{Kr}$ inventory calculated with the ${ }^{10} \mathrm{~B}$ concentration in the capsule updated at every 5-day interval (Frequent B-10 update) compared with those predicted by a polynomial fit of the inventory calculated four irradiation days after each outage with ${ }^{10} \mathrm{~B}$ concentration updated only during outages

12. Percentage recovery of input value as a function of gamma-ray energy for the SYNTH model

13. Comparison of the estimated sample + background spectrum resulting from a single particle failure (upper curve) with the expected background from heavy metal contamination (bottom curve)

14. An annotated section (Channels 1000 to 2000) of the spectral comparison of the previous figure

15. Figure depicting the net count rate distributions for the detection sensitivity work......................20

16. The shield for the $\mathrm{NaI}(\mathrm{Tl})$ gross radiation detector will be cast from bismuth

17. A sketch of one of the FPMS stations showing the gross monitor shield and the HPGe detector and shield

18. Net counts per second versus $1 / \mathrm{x}^{2}$ for two amplifier-to-Mutiport II configurations

19. Scanned image of the gross monitor response to the first particle failure from the NPR-1 Test. 
20. The simulated response of the AGR-1 gross detector system to the failure of a single particle at 244 EFPD

21. The simulated response of the AGR-1 gross detector system to the failure of the 251st particle at 244 EFPD...

22. Estimated total activity per $\mathrm{cm}^{3}$ at a given gross detector as a function of test duration.

\section{TABLES}

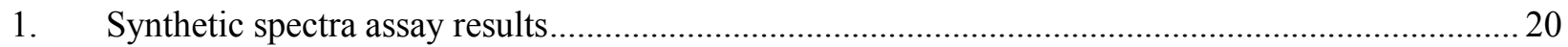

2. Calculated system sensitivity parameters compared with the expected release from a single TRISO particle failure at 764 EFPD of irradiation 


\section{Design and Expected Performance of the AGR-1 Fission Product Monitoring System}

\section{INTRODUCTION}

The US Department of Energy (DOE) is embarking on a series of tests of coated-particle reactor fuel for the Advanced Gas Reactor (AGR). As one part of this fuel development program, a series of eight (8) fuel irradiation tests are planned for the Idaho National Laboratory's (INL's) Advanced Test Reactor (ATR). The first test in this series (AGR-1) will incorporate six separate capsules irradiated simultaneously, each containing about 51,000 TRISO-coated fuel particles supported in a graphite matrix and continuously swept with inert gas during irradiation. The effluent gas from each of the six capsules must be independently monitored in near real time and the activity of various fission gas nuclides determined and reported.

The effluent from each test capsule of the AGR-1 experiment will be monitored by a detector system consisting of a gamma-ray spectrometer and a gross radiation detector. This collection of radiation measurement systems will be known as the AGR-1 Fission Product Monitoring System (FPMS). Proper design and functioning of the FPMS is critical to the success of the AGR-1 fuel test experiment.

The AGR-1 FPMS will be one of a succession of on-line fission product monitoring systems designed, installed and operated by scientists from the Reactor and Nuclear Physics group at the Idaho National Laboratory (INL) and its predecessor organizations. One of the earliest (1977) implementations was an unattended monitor installed in the Advanced Test Reactor (ATR) to monitor stack effluents in real time. An updated version of this unit still provides useful information on the stack releases. The success of the Severe Fuel Damage test series conducted in the Power Burst Facility (PBF) in the early to mid 1980s was largely due to a fission product detection system designed by this organization. ${ }^{i}$ Members of the Reactor and Nuclear Physics group have also designed, implemented and operated fission product monitoring systems for in-reactor gas-cooled fuel experiments including the New Production - Modular High Temperature Gas-coolant Reactor (NP-MHTGR) fuel performance test 1A (NPR-1A) ${ }^{\mathrm{ii}}$ and as well as other ongoing experiments. The design of the AGR-1 FPMS described in this work borrows heavily from a previously implemented and very successful monitoring system.

The AGR-1 Irradiation Test Specification ${ }^{\text {iii }}$ includes the following requirements pertinent to the FPMS:

- $\quad$ Each of the six test capsules are to be independently monitored for fission product gas release.

- $\quad$ Sensitivity of the FPMS shall be sufficient to detect every individual particle failure, up to and including the first 250 failures, from each capsule.

- $\quad$ Transit time of the sweep gas from each capsule to the FPMS shall be $<25$ minutes.

- $\quad$ Total radiation level of the sweep gas from each capsule shall be measured and recorded continuously during irradiation.

- $\quad$ Concentrations of at least ${ }^{85 \mathrm{~m}} \mathrm{Kr},{ }^{87} \mathrm{Kr},{ }^{88} \mathrm{Kr},{ }^{133} \mathrm{Xe}$, and ${ }^{135} \mathrm{Xe}$ shall be measured in the sweep gas from each capsule and recorded at least daily during irradiation.

- $\quad$ All test data shall be backed up and stored in separate facilities at least daily. 
This document describes the AGR-1 FPMS and presents calculations indicating that this design will meet the pertinent test requirements.

\section{DESIGN OVERVIEW}

\subsection{Introduction}

The FPMS will incorporate seven (7) individual monitoring systems - one for each of the individual capsules effluent lines, and one unit that can monitor any individual effluent line or any combination of the six lines. The seventh monitor is primarily provided as a back up unit capable of providing effluent line monitoring if any primary monitor systems fails. Each monitor consists of a high purity germanium (HPGe) detector-based gamma-ray spectrometer and a sodium iodide [NaI(Tl)] scintillation detector-based total radiation detector (often termed the "Gross" radiation detector). These units will be located in the ATR 2C Secondary cubicle. In addition, a grab sample station will be provided to allow sampling of the effluent from each or any combination of the six test capsules to be sampled for off-line analysis. An overview sketch is provided in Figure 1. Complete details are provided in a drawing package. ${ }^{\text {iv }}$

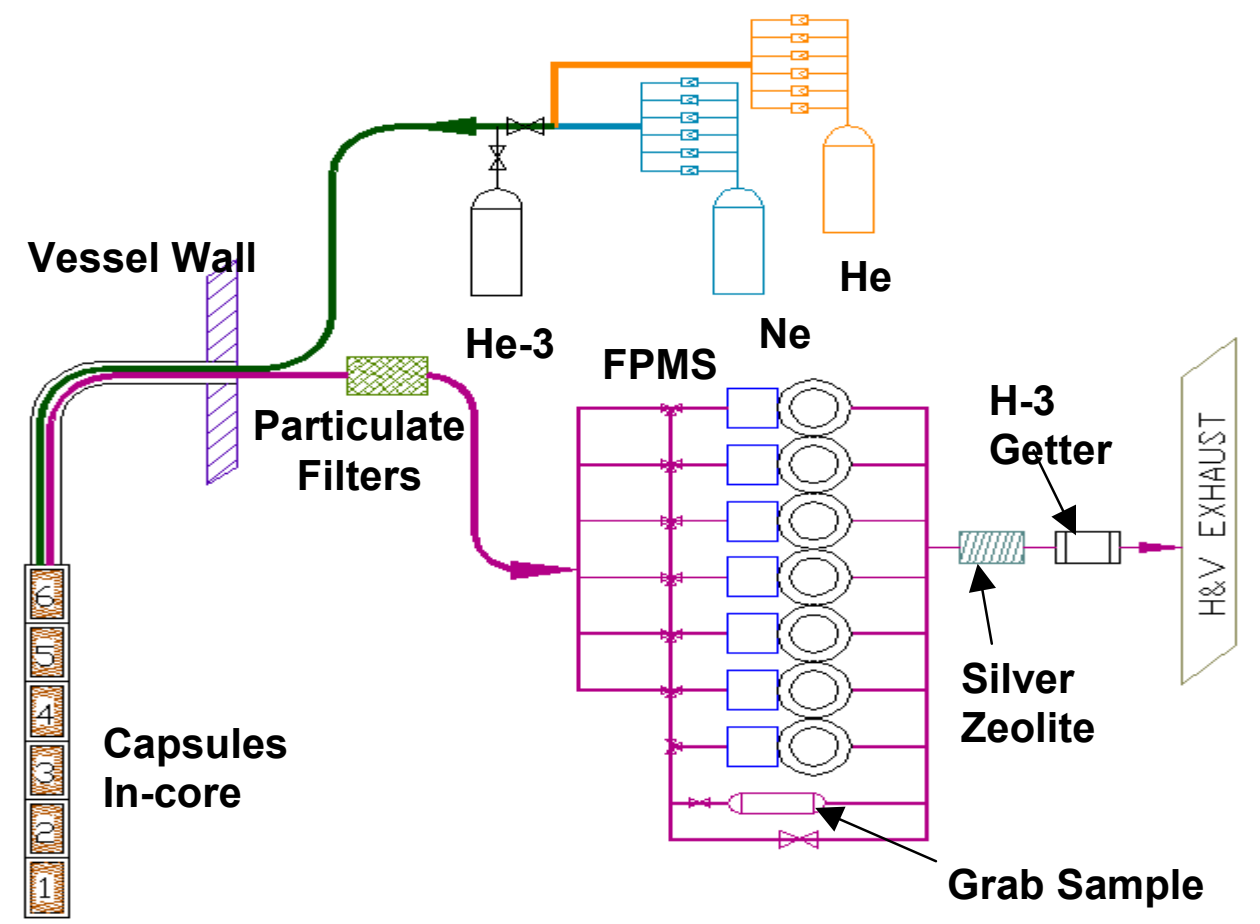

Figure 1. Overview depiction of the sampling system flow path.

The sweep gas from each test capsule is routed via sampling lines to the monitoring station associated with that capsule. Sample transit times from capsule to FPMS at the normal test flow rate of $30 \mathrm{~cm}^{3} / \min \left(0.5 \mathrm{~cm}^{3} / \mathrm{s}\right)$ are estimated to be about 2.5 minutes. For certain short duration periods of high reactor power operation, the sweep gas flow rate may be decreased to as little as $2 \mathrm{~cm}^{3} / \mathrm{min}\left(0.033 \mathrm{~cm}^{3} / \mathrm{s}\right)$ increasing the sample transit times to as much as 36 minutes. Although this transport time exceeds that allowable in the test specification preliminary calculations ${ }^{\mathrm{v}}$ indicate that all but the shortest-lived fission gases remain detectable at the longer transport time, thus it should be acceptable. The sample lines, valves, and filters are predominately contained in the $2 \mathrm{C}$ Primary cubicle. The sample lines have only 
short, shielded segments in the $2 \mathrm{C}$ secondary cubicle. These short segments run through the gross detector monitoring station and then into the HPGe spectrometer shield.

Each gross detector monitoring station (seven will be implemented) incorporates a $\varnothing 25 \mathrm{~mm} \times$ $25 \mathrm{~mm}$ thallium-activated sodium iodide [ $\mathrm{NaI}(\mathrm{Tl})]$ scintillation detector viewing a $25 \mathrm{~mm}$ long segment of the capsule effluent line just before its entry into the HPGe spectrometer shield. The scintillation detector counting rate is monitored using a computer-controlled multichannel scaler (MCS). Further details on the gross radiation monitors are provided in Section 4 of this document.

The HPGe spectrometer stations are designed to mimic a previous successful implementation. The detector configuration, shield and table design, and the sample container are all taken from the archived drawings. The sample container, also known as the sample "trap", will be the existing warm trap design from this implementation. The shield will be a specially-modified commercially-available HPGe spectrometer shield. ${ }^{\mathrm{vi}}$ Figure 2 provides a sketch of the proposed configuration while Figure 3 shows a photograph of six similar spectrometers presently installed in the ATR. The proposed AGR-1 HPGe spectrometer installation will be similar. More details are presented in Section 3.0 of this document.

The gamma-ray spectrometers and gross detectors are supported by commercial electronic components modified to incorporate the INL-patented technique of pulse injection with subsequent removal. ${ }^{\text {vii }}$ The spectrometer electronics are identical to those used successfully in previous measurement systems and their performance characteristics are well known. The gross detectors are supported by commercial amplifiers with the outputs scaled using a Canberra Multiport II multi-input multichannel scaler (MCS). This configuration has been tested for input rate performance and the results are presented in Section 4 of this document.

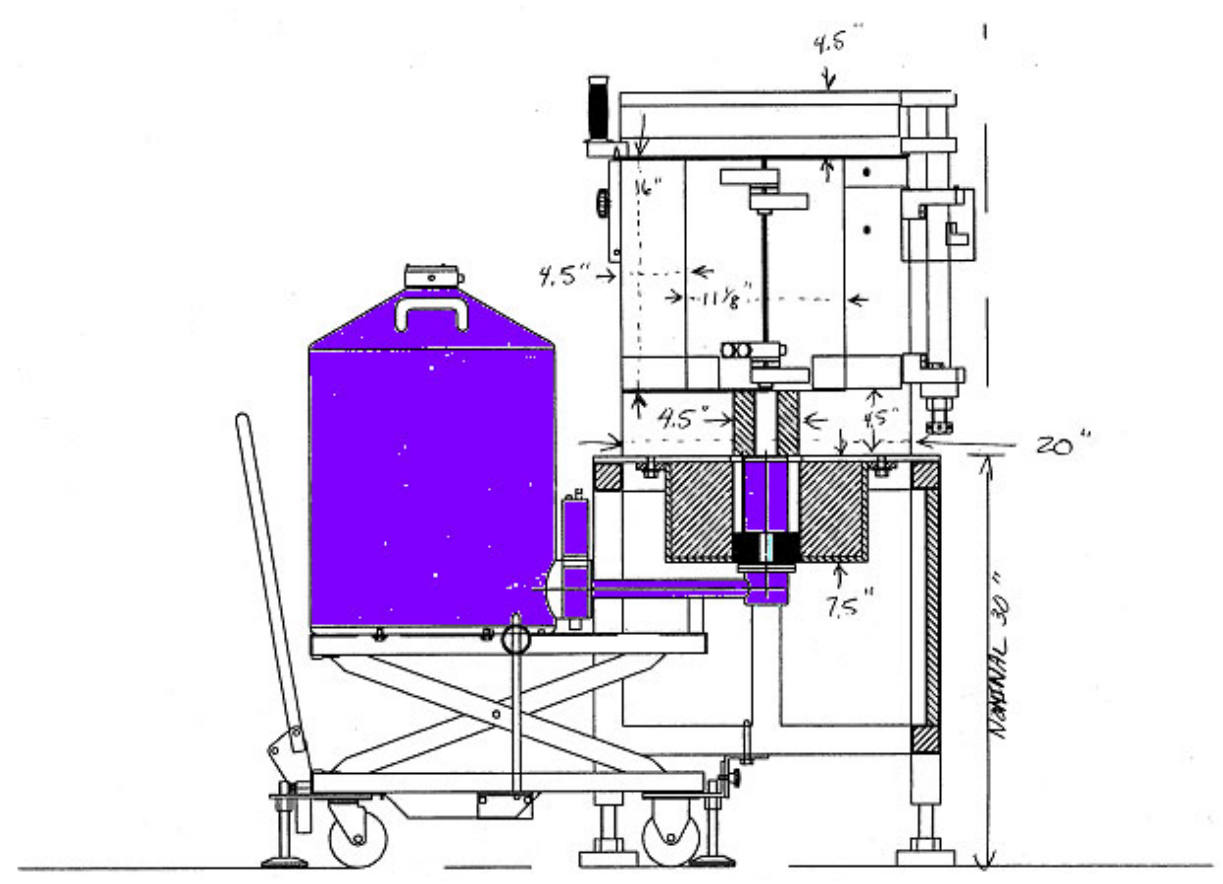

Figure 2. A sketch of the FPMS shield and detector arrangement. The detector has been filled in to highlight its position. The detector views a sample chamber (not shown) through the shaded collimator. The sample chamber is located at the bottom inside of the shield. 


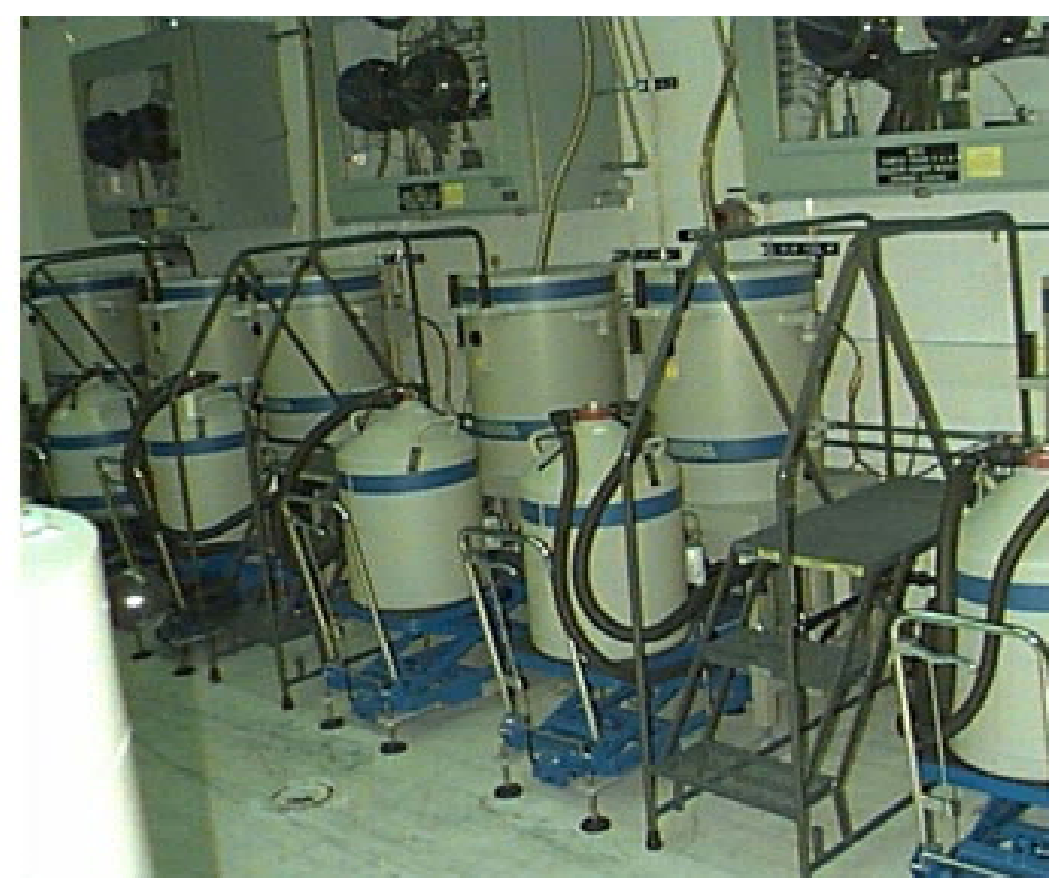

Figure 3. Six gamma-ray spectrometers presently installed in the ATR. These are similar to those planned for AGR-1.

\subsection{Hardware Overview}

The schematic below (Figure 4) shows the hardware components of the FPMS and the flow of control and data communications between the different component sub-systems. Each of the seven detector systems will consist of an HPGe detector and a NaI(Tl) detector. These two detector sub-systems will be housed in radiation shields to reduce the ambient background detected.

The HPGe output signals will be processed through an amplifier and then input to an analog-to-digital converter (ADC). The digitized ADC output will increment the value in an address of the histogramming memory contained in a Canberra Model 556A Acquisition Interface Module (AIM) MCA. The AIM MCA is computer controlled with all control communications and data transfers performed over a standard Ethernet connection. The AIM is programmed to initiate a measurement cycle of a fixed time period. Its internal processor performs the MCA functions as well as keeping track of measurement times. At the end of the set acquisition time, the AIM holds the histogrammed spectral data until requests are received from the host computer to transmit the accumulated spectral information, clear the memory and restart a new acquisition. A transmit, reset, and restart cycle can be accomplished in less than 1 second. The AIM modules were selected for the AGR-1 application because of their successful implementation in a number of measurement systems at the INL. Also the AIMs and the chosen ADCs are compatible with the patented pulser injection with subsequent removal technology ${ }^{\text {vii }}$ developed at the INL to monitor spectrometer dead-time and energy calibration. Each AIM module will service two ADC's and are rated up to a $1 \mathrm{MHz}$ data rate. 


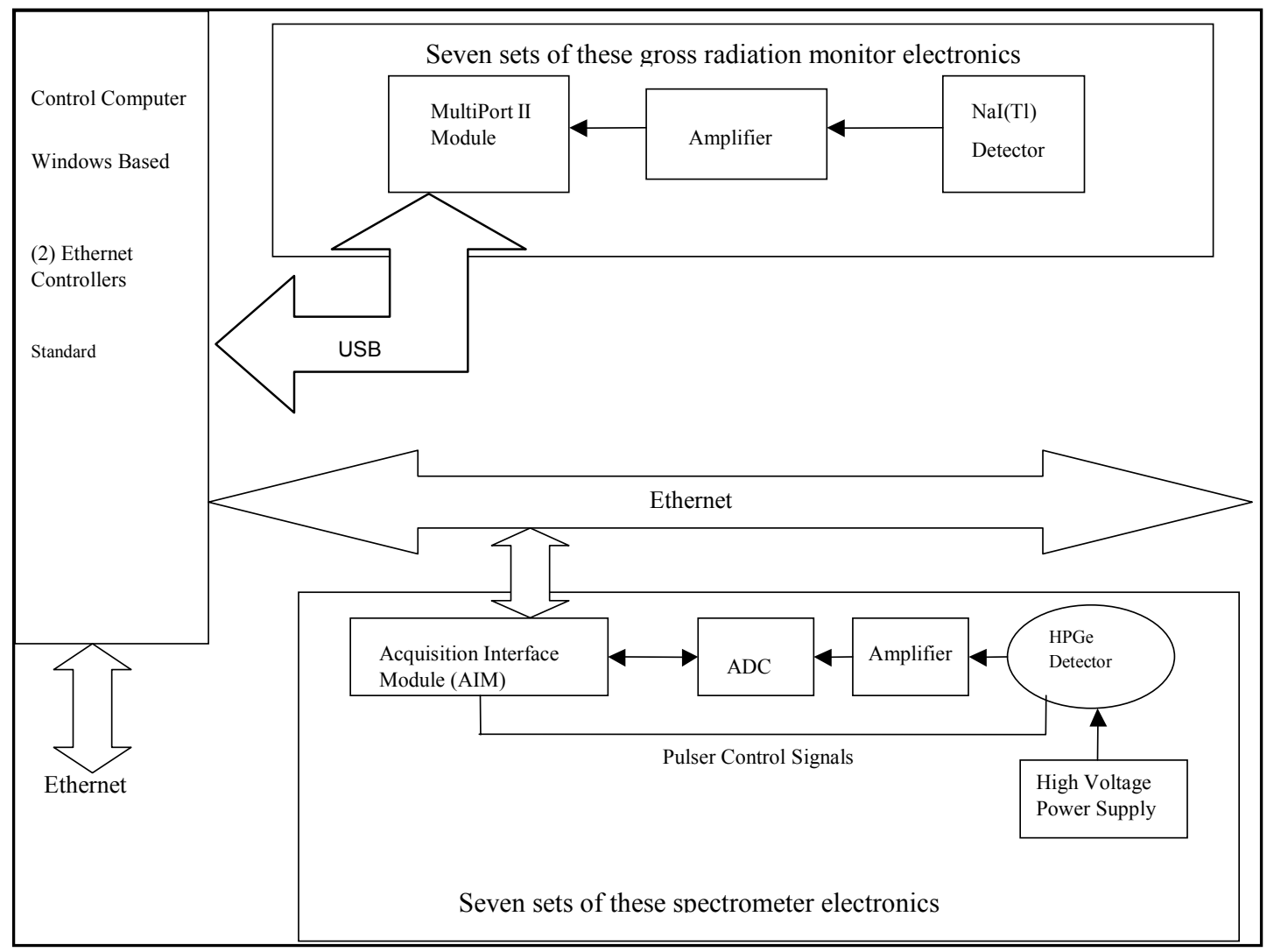

Figure 4. Hardware components of the Fission Product Monitor System (FPMS) and the flow of control and data communications between the different component sub-systems.

A shielded $\mathrm{NaI}(\mathrm{Tl})$ scintillation detector viewing a section of sample line will be used to measure changes in the radiation field in each capsule's effluent. The count rate information obtained from the $\mathrm{NaI}(\mathrm{Tl})$ detector will be recorded with a MCS concurrent with the HPGe gamma-ray spectral measurement. The channel dwell time of the MCS will be selected so that the time interval represented by the full range of the MCS spectrum will match the measurement time used to collect spectral data for the effluent gas.

The MCS selected for this operation is the Canberra Multiport II. The Multiport II will be controlled and accessed through a Universal Serial Bus (USB) using the same Genie 2000 virtual data manager (VDM) that is used to communicate with the AIM.

The Canberra AIM modules will control and acquire data from two ADC's each so that only four AIM modules will be needed for the primary measurement system. The Canberra Multiport II module comes with between 1 to 6 MCS channels. The four MCS option for the Multiport II modules was selected for the FPMS to provide the seven inputs required and to provide one spare channel.

Both the AIM and Multiport II units will continue to collect data if there is a loss of communications with the host control computer. At the end of the designated measurement time, the units will hold the collected data until it is requested by the host computer and a new measurement is started. 


\subsection{Software}

The operation of the seven HPGe spectrometer and gross monitoring systems will be controlled from a common host computer. The control and data transfer communications will be carried out over a private network subnet made up of the host computer and the intelligent multi-channel analyzers. Each of the seven measurement stations will have individual MCA's for the HPGe spectrometer and the gross monitor. The host computer will have a second network interface connected to the INL laboratory network to allow remote monitoring of the FPMS and to backup the collected data to remote storage. The individual monitoring systems will be operated continuously throughout the AGR fuel irradiations. The operation of the individual monitoring stations will follow a set sequence of operations. The first step of this sequence is the start of an HPGe spectrometer and gross monitor measurement. These measurements will run for a set period of time determined by the experimental staff based on test conditions. At the end of the set measurement time, the experiment data will be collected from the system's MCAs and MCSs by the control program and saved to disk. Transmission times will be less than one second, and after the data has been saved, data collection will restart. The acquired gamma-ray spectral data will be sent to the analysis program where the quantities of certain select fission products in the effluent gas will be determined. Changes in the radionuclide quantities and in the gross count rates will be used to indicate the failure of AGR fuel particle. The collected spectral data, gross count rate data, and analysis results will be saved to the computer hard disk for later archival storage. This information will be periodically backed up to a remote file system and archived to some long-term storage media. Each spectrometer/gross monitor system can be operated independently depending on conditions.

The control system software is based on a program developed for manually controlling the seven spectrometers used in a previous experiment. The program has a standard Windows user interface, and it is designed to operate without continual user intervention and monitoring. The communications between the control program and the Canberra MCAs are carried out through a proprietary Canberra Virtual Data Manager (VDM). Commands to the AIM-based MCAs and readout of the data stored in the AIM-based MCAs are via an ethernet connection between the host computer and the AIM units. Control commands and data readout from the Multiport II MCS units is via a USB connection, in order to avoid some protocal conflicts detected when both the Multiport IIs and the AIMs were connected via Ethernet. The VDM handles all of the low-level communications between the commands sent out by the control program and the MCA/MCS hardware. The control program communicates to the VDM using the proprietary Canberra Genie 2000 Programming Library.

The interface and control software will perform the following functions:

1. Provide the user interface for the operation of a HPGe spectrometer and $\mathrm{NaI}(\mathrm{Tl})$ gross monitor detectors for each of the six fuel capsules and for the seventh reserve spectrometer. The interface will provide the capability to modify the operation of any of these peripherals in real time.

2. The software shall allow the user to display the contents of any spectrum stored on disk or display the real-time data acquisition of any single pulse-height spectrum.

3. The parameters used to control the spectrometers will be saved in a disk file. The values of these parameters will be restored from the disk files in case of a power outage. This function will allow the spectrometers to be returned to normal operation in the shortest amount of time without operator intervention.

4. Each HPGe spectrometer/NaI(Tl) gross monitor detector will have the ability to be placed off line without adversely affecting the operation of the other spectrometers. 
5. All collected spectra will be analyzed and initial radionuclide concentrations computed. The concentration values will be made available in a format that will allow the data to be readily imported into a spreadsheet. The initial analysis will be carried out by the same software used for similar experiments.

6. In order to allow maximum independence between the operation of each of the spectrometers, a separate execution thread will be run for each HPGe spectrometer/NaI(Tl) detector system. A failure of one or more of the spectrometer hardware or execution threads will not cause the execution of the other spectrometer systems to fail.

7. The normal operation of the monitors will be a continuous loop of collecting and then analyzing the resulting spectrum. The length of data collection will be a user-modifiable parameter. All spectral and corresponding analysis files will be saved on disk and shared with other computers.

8. The software shall save the MCS spectra showing the $\mathrm{NaI}(\mathrm{Tl})$ detector count rates as a function of time. The time range of the spectra will be matched to the sample measurement times used for the corresponding HPGe spectroscopic spectra. The MCS spectra will be stored on disk in the same format used by the HPGe spectrometer data and can be examined using the same analysis software.

9. The spectra collected will be stored separately on disk depending on the type of measurement. The spectra will be stored with unique file names that identify the spectrometer as well as the time and date of the measurement. In addition, a spectral header provides full identification of the acquisition details.

10. The radionuclide analysis listing will be saved and the filenames will make use of the same format used by the corresponding spectrum files.

The main process thread of the software will provide the control interface to the user for setting the sequencing and timing of the fission product measurements. The control interface will also provide feedback to the operator of any error conditions that are detected by the individual spectrometer processing threads (Figure 5). The type of errors to be reported include communications problems between the control computer and the AIM MCA and Multiport II MCS modules, excessive spectrometer count rates, problems with the archiving of the measurement data, or problems with the online analysis tasks.

The operational history of the FPMS will be saved in a log file generated by the control software. This log file will record the start and stop times for the different spectrometers, the location and file names of the archived spectral and gross monitor data, and any error conditions that occur. This log file will be saved along with the measurement data to provide a permanent record of the FPMS operation.

The main process thread will start the individual spectrometer control processes and will initiate the start and stopping of the effluent sample measurements (Figure 6). The spectrometer control processes will initiate the required measurement, save the resulting data, and start the appropriate on-line analysis task. 


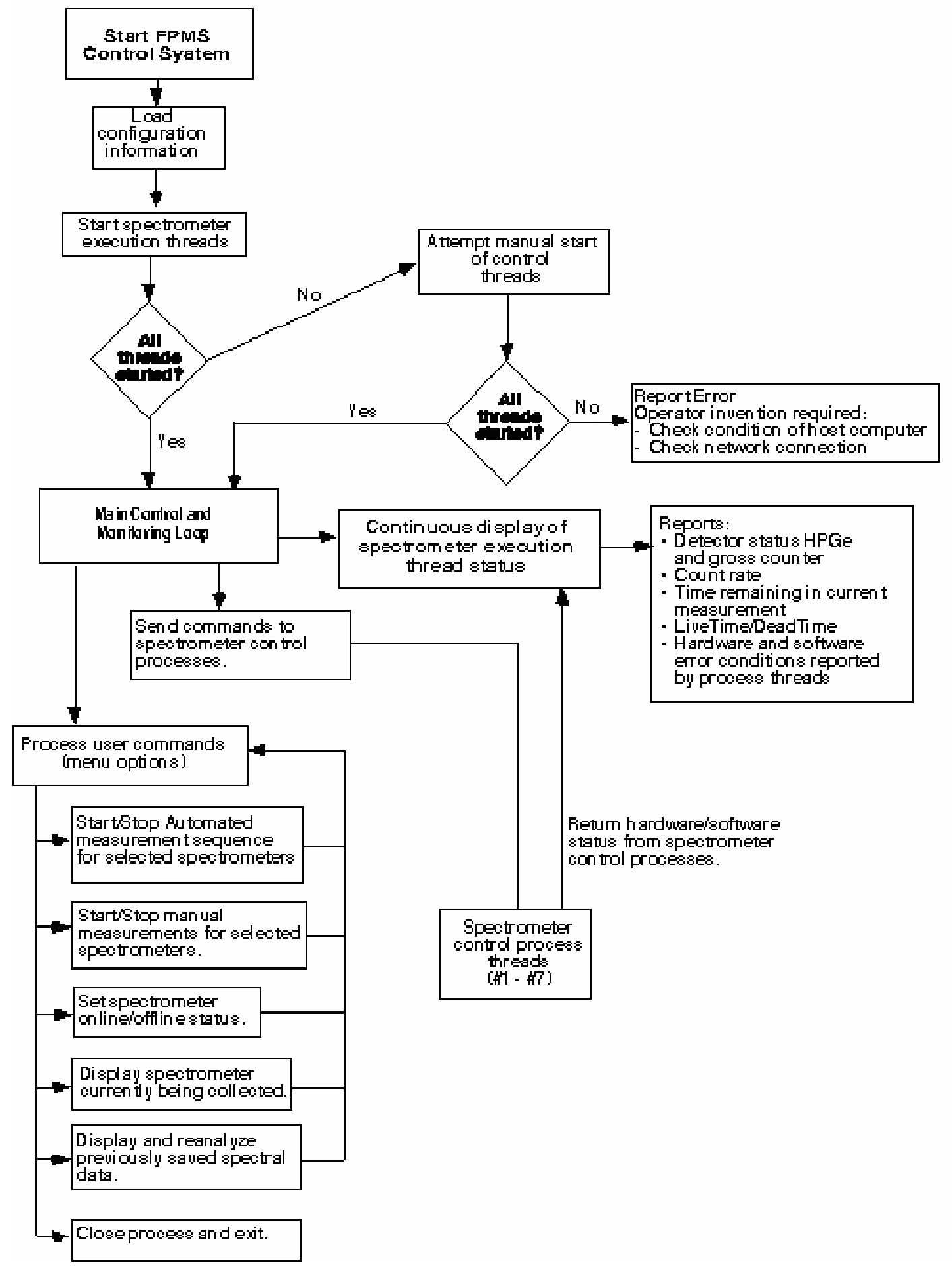

Figure 5. FPMS Control System flow diagram. 


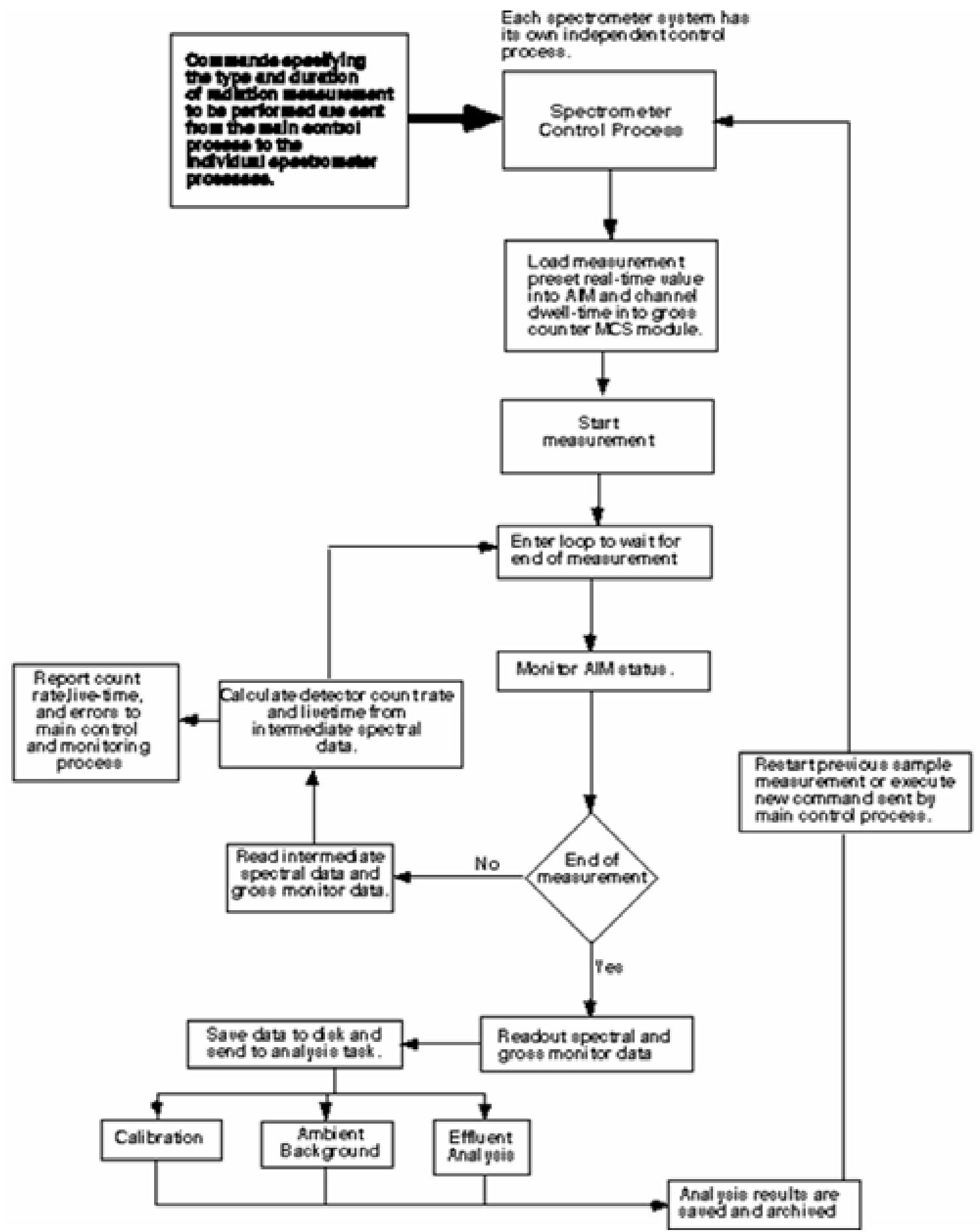

Figure 6. FPMS spectrometer control process flow diagram. 


\section{FPMS HPGE SPECTROMETERS}

\subsection{Equipment and Design}

\subsubsection{Detectors and Shields}

The HPGe detectors used in the AGR-1 FPMS will be similar to those employed in a presently implemented fission product monitoring system. These are nominally $12 \%$ relative efficiency closed-end coaxial P-type detectors housed in a special cryostat. ${ }^{\text {vii }}$ The cryostat configuration mates with the shield configuration $^{\mathrm{vi}}$ and provides for excellent detector shielding. The detector views the sample trap from the bottom through a collimator in the bottom of the shield. Three different collimator diameters are available to control the gamma-ray fluence reaching the detector face. Detector signals are extracted and shaped by a Canberra 2002C preamplifier. A sketch of the assembled detector and shield arrangement is provided in Figure 7. The capsule effluent is carried through tubing that passes through a gross radiation monitoring assembly attached to the rear of the HPGe shield, then into a nominally $50 \mathrm{~cm}^{3}$ sample chamber volume viewed from below by the HPGe detector through a replaceable collimator. The relative positioning of these components is depicted in Figure 8 while a set of photographs in Appendix A shows details of the spectrometer components under testing in our laboratory.

\subsubsection{Spectrometer Electronics}

The AGR-1 FPMS spectrometers will use electronics similar to those implemented for previous systems. Figure 9 presents a block diagram of an FPMS spectrometer. Detector signals are amplified in standard spectroscopy amplifiers (C2026), processed in analog-to-digital converters (ADCs) modified to include pulser injection control and event separation (C8715), vii and the ADCs provide input to a set of AIMs (C556A) that acquire the spectral data. On completion of an acquisition interval, the acquired spectra are transmitted (via Ethernet) to the control computer. Transmission times are less than one second.

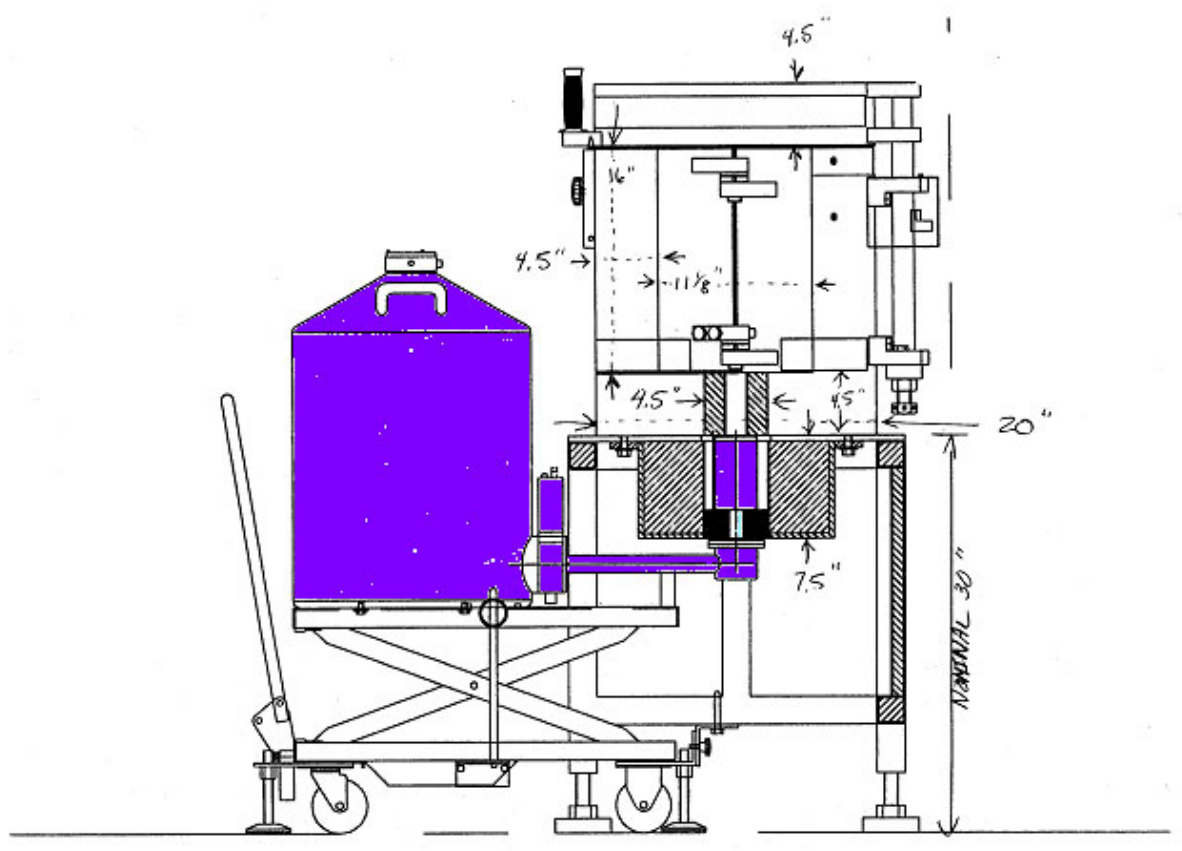

Figure 7. Sketch of the HPGe detector and shield. The detector components have been shaded for clarity. 


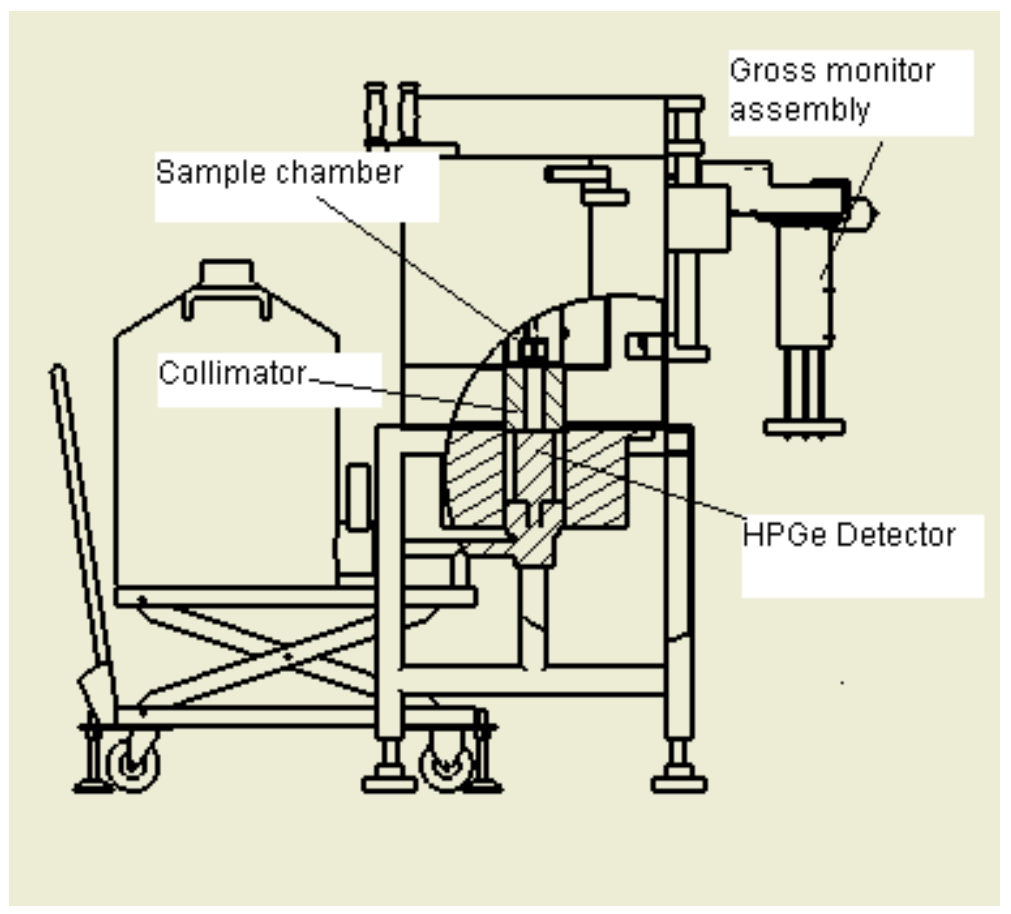

Figure 8. Partial cutaway sketch of an FPMS station showing the relative positioning of certain components.

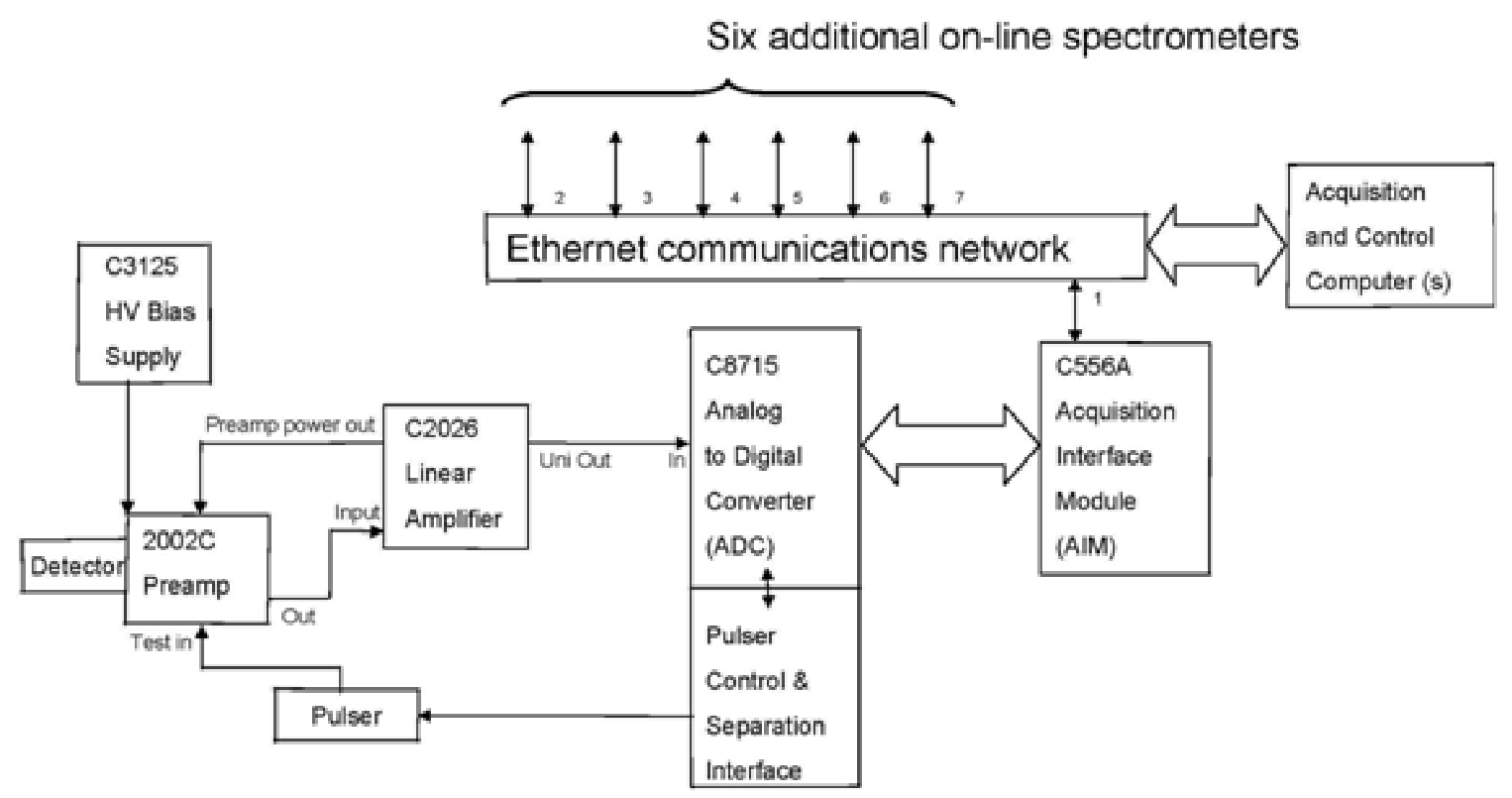

Figure 9. Block diagram of the FPMS spectrometer electronics. 


\subsubsection{Software and Control}

The spectral data from each HPGe spectrometers will be acquired in Canberra 556A AIM modules. A function of the FPMS control program is to start the collection of data by the AIM MCA for a specified real-time interval. The control software will periodically monitor the operation of the AIM to calculate detector count rates and report any problems due to hardware or software errors. At the end of the preset measurement time, the control program will read out the histogram data from the AIM MCA. The acquired spectroscopic data will be saved to a disk and will be named to identify clearly which spectrometer acquired the data and the time and date that the measurement was started. Acquisition of a new spectrum will be started automatically. The initial analysis of the data will be done automatically following each measurement and the results, as well as the raw spectral data, written to a file for storage. The will be performed using the PCGAP analysis software package ${ }^{\mathrm{ix}}$ and will determine and report the quantities of certain fission product gases present in the effluent gas. All analysis results will be stored for later review. The concentration results for certain fission products selected by the FPMS staff will be stored in an appropriate file for near real-time review. Results and acquired spectral data will be archived for later qualification by a trained professional.

\subsection{Expected Releases and Detection Sensitivity}

\subsubsection{General}

An important requirement of the AGR-1 FPMS is that it must be sufficiently sensitive to enable detection of the failure of a single fuel particle at any time during the test. (In our implementation, the task of "counting" several [up to 250] successive particle failures is the job of the gross radiation detection system [see Section 4]). Isotopic quantification of the noble gas release concentrations is required to support determination of fission gas release-to-birth ratios. ${ }^{\mathrm{x}}$ To ensure that our spectrometer design meets this specification requires an estimate of the fission gas activity released by a failed particle (the source term) and an estimate of the detection sensitivity of the spectrometer system.

Capsule fission particle inventory data have been calculated using a code that couples Monte Carlo calculations with ORIGEN2 inventory calculations. ${ }^{\mathrm{xi}}$ The Monte Carlo component is required to determine the depletion of the ${ }^{10} \mathrm{~B}$ in the $\mathrm{B}_{4} \mathrm{C}$-impregnated graphite used as a burnable poison in the AGR-1 capsule design. ${ }^{\text {xii }}$ This code is termed "Monte Carlo with ORIGEN2" or MCWO. These inventories were used along with standard calculational formulations ${ }^{\text {xiii }}$ to predict steady-state fission product release rates both for the activity released upon failure of a single fuel particle and for the background from initially failed particles and heavy metal contamination of the particles and graphite above which the particle releases must be detected.

The detection sensitivity of a gamma-ray spectrometer system is dependent on the background (continuum) above which the signal must be detected, the absolute photopeak efficiency of the detection system, and the counting time. Because of Compton scattering of gamma-rays, each spectral background is profoundly affected by the mix and quantity of gamma-ray emitting nuclides in the viewed sample. Consequently, the detection efficiency for isotopes in a given mix is best derived from either actual or synthetic spectra since these reproduce the expected spectral continuum. In this work, an accepted and widely used spectral synthesis program, $\mathrm{SYNTH},{ }^{\text {xiv }}$ was used to construct synthetic gamma-ray spectra from input data.

Preliminary estimates of the AGR-1 FPMS spectrometer detection sensitivity have been reported. ${ }^{\mathrm{v}}$ The work reported in this section uses the same calculational approach as the preliminary work, but it uses inventory and source term data updated to reflect the present test planning. 


\subsubsection{Source Term Estimates}

3.2.2.1 Article Inventory Estimates. A steady state release model was used to predict the background from the expected heavy metal contamination and to predict the increase in fission gas activity expected at an FPMS detector upon particle failure. The fission gas inventory values were those reported by $\mathrm{Chang}^{\mathrm{xv}}$ for the present test configuration with $5 \% \mathrm{~B}_{4} \mathrm{C}$ in the graphite of the top capsule, $6 \%$ $\mathrm{B}_{4} \mathrm{C}$ in the bottom capsule, $7 \% \mathrm{~B}_{4} \mathrm{C}$ in the remaining four test capsules, and a $240^{\circ}$ Hafnium shroud on the near-core side to even out the exposure between fuel compact stacks. For these calculations, each of the three fuel stacks were divided into 48 calculational nodes. Of the 144 inventory results, those from node 69 (capsule 4 stack 2) were chosen as a near maximum inventory and used in these source term calculations. To decrease the data handling, initial inventory calculations were performed with the ${ }^{10} \mathrm{~B}$ concentrations updated only during the outages between irradiation cycles. Inventories were calculated at 4-day irradiation intervals, but because of the timing of the ${ }^{10} \mathrm{~B}$ depletion updates, calculated inventories late in an irradiation cycle are in error. This is particularly true during the early cycles when ${ }^{10} \mathrm{~B}$ concentrations are highest. Because of this effect, only those values from the first four-day irradiation period after an outage were presumed to be correct. Since this evaluation is limited to fission gas nuclides with a half live less than 12 hours, this is a reasonable assumption, and it has been verified by comparison to more detailed calculations discussed later in this section. Figure 10 plots the ${ }^{85 \mathrm{~m}} \mathrm{Kr}$ inventory in Curies $/ \mathrm{cm}^{3}$ of capsule volume calculated in two ways. MCWO is the data presented at 4-day intervals throughout the test duration with ${ }^{10} \mathrm{~B}$ concentrations updated only during outages between cycles. The anomalous increases during the early outage periods are the result of ${ }^{10} \mathrm{~B}$ concentration updates that deplete the ${ }^{10} \mathrm{~B}$-poison only during these outages. The dashed line (Poly.) is the result of fitting the MCWO-calculated inventory at 4 days into each irradiation cycle with a polynomial to predict a more correct intracycle inventory.

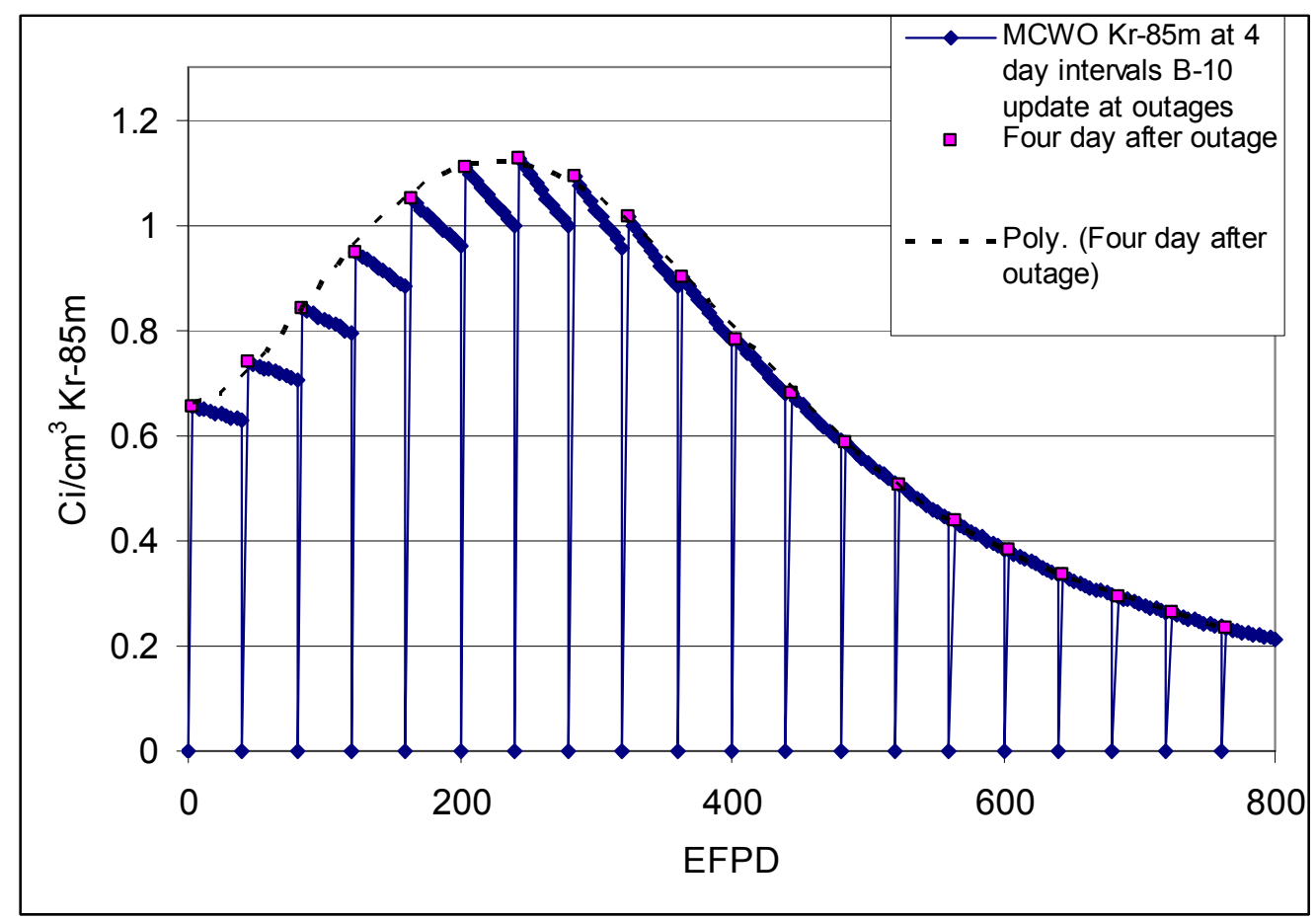

Figure 10. Inventory data for ${ }^{85 \mathrm{~m}} \mathrm{Kr}$ presenting the calculated MCWO results and the fitted values used in this work. 
Certain of the early AGR-1 inventories were recalculated using MCWO calculations that recomputed both the ${ }^{10} \mathrm{~B}$ depletion and the fission product inventories at 5-day intervals. In order to confirm the validity of the procedure (described in the previous paragraph) of using only the results from the first four days of each irradiation period and ${ }^{10} \mathrm{~B}$ concentration updates only during outages, results for the ${ }^{85 \mathrm{~m}} \mathrm{Kr}$ inventory calculated in the exhaustive calculation with frequent 5-day updates of the ${ }^{10} \mathrm{~B}$ concentrations were compared with those computed from a polynomial fit of the inventories calculated at 4 days into each irradiation cycle for certain of the early irradiation cycles (when ${ }^{10} \mathrm{~B}$ concentrations are most important). Figure 11 presents this comparison. The results differ by less than $4 \%$. This comparison indicates that the inventory predictions computed from fitting the inventory values on the fourth day of each irradiation period from calculations with a B-10 update only during outages provides a reasonable estimate of the true inventory versus time.

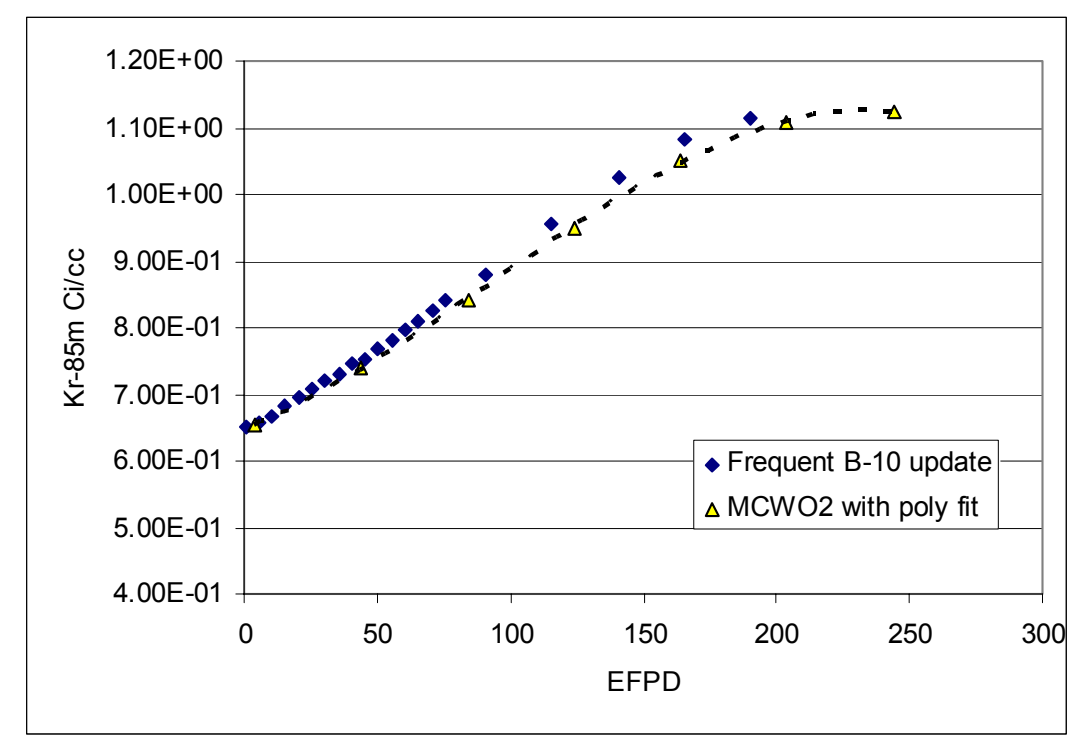

Figure 11. A comparison of the ${ }^{85 \mathrm{~m}} \mathrm{Kr}$ inventory calculated with the ${ }^{10} \mathrm{~B}$ concentration in the capsule updated at every 5-day interval (Frequent B-10 update) compared with those predicted by a polynomial fit of the inventory calculated four irradiation days after each outage with ${ }^{10} \mathrm{~B}$ concentration updated only during outages.

Inventories of all of the fission gas nuclides were predicted from similar polynomial fits to the MCWO data set. The activity concentrations in Curies $/ \mathrm{cm}^{3}$ predicted from each polynomial fit were converted to average Curies per fuel particle by dividing by 1410 particles per $\mathrm{cm}^{3}$. These particle inventories are used in the next section.

3.2.2.2 Cell Release Rates. Fission gas releases can be from beginning of life heavy metal contamination on the particles and compacts, initially failed particles, or they can be from the failure during the test of previously intact fuel particles. For the balance of this discussion, the term "heavy metal contamination" will be used to denote heavy metal contamination of the particles and compacts and initially failed particles, those features that contribute to a fission gas release background above which any test-induced particle failures must be detected.

Calculations in this EDF are restricted to fission gas nuclides with decay half lives of $\leq 12$ hours. This is to ensure that the fission gas activity inventories (in Bq) are equal to their birth rates. All calculations assume steady state. 
Capsule release rates for a given nuclide are calculated as ${ }^{\text {xiii }}$

$R=B * N_{\text {particle }} *\left\lfloor\left(F_{\text {particle }} *(R / B)_{\text {particle }}\right)+\left(F_{H M} *(R / B)_{H M}\right\rfloor\right.$

where

$$
\begin{aligned}
& \mathrm{R}=\text { the capsule release rate in atoms } / \mathrm{s} \\
& \mathrm{B}=\text { the birth rate of the nuclide in atoms/s per particle. Assumed equal to the } \\
& \text { inventory decay rate in } \mathrm{Bq} \text { (Curies*3.7E10) } \\
& \mathrm{N}_{\text {particle }}=\text { the number of particles in a capsule (51,360 particles) } \\
& \mathrm{F}_{\text {particle }}=\text { the particle failure fraction, assumed initially to be zero. } \\
& \text { Failure of a single particle gives } \mathrm{F}_{\text {particle }}=1.95 \mathrm{E}-05 \\
& (\mathrm{R} / \mathrm{B})_{\text {particle }}=\text { release to birth rate ratio for a failed particle } \\
& \mathrm{F}_{\mathrm{HM}}=\text { the heavy metal contamination expressed as an initial failure fraction. The fuel } \\
& \text { specifications require that } \mathrm{F}_{\mathrm{HM}} \text { be less than 1.0E-04 (95\% CL). } \\
& (\mathrm{R} / \mathrm{B})_{\mathrm{HM}}=\text { the release-to-birth rate ratio for heavy metal contamination }
\end{aligned}
$$

Release rate to birth rate ratios are estimated using the Booth equivalent sphere gas release model and semiempirical reduced diffusion coefficients. ${ }^{\text {xiii }}$ The formulation is:

$(\mathrm{R} / \mathrm{B})=(3 / \mathrm{X}) *[\operatorname{coth}(\mathrm{X})-(1 / \mathrm{X})]$

where

$$
\begin{aligned}
& \mathrm{X}=\left[\lambda / \mathrm{D}^{\prime}\right]^{1 / 2} \\
& \lambda=\text { the decay constant of the nuclide of interest }\left(\mathrm{s}^{-1}\right) \\
& \mathrm{D}^{\prime}=\text { the reduced diffusion coefficient for the release scenario }\left(\mathrm{s}^{-1}\right)
\end{aligned}
$$

$\operatorname{coth}(\mathrm{X})=[\exp (\mathrm{X})+\exp (-\mathrm{X})] /[\exp (\mathrm{X})-\exp (-\mathrm{X})] \approx 1.0$ for $\mathrm{X}>>1.0$

Different values for the reduced diffusion coefficient are recommended depending on the nuclide of interest and the nature of the exposed heavy metal. For heavy metal contamination:

$\mathrm{D}^{\prime}=3.0 \mathrm{E}-05 *[\exp (-1.06 \mathrm{E}+05 /(8.314 * \mathrm{~T}))]$ for krypton nuclides

and

$\mathrm{D}^{\prime}=1.7 \mathrm{E}-07 *[\exp (-7.86 \mathrm{E}+04 /(8.314 * \mathrm{~T}))]$ for xenon nuclides 
For failed particles in the temperature range of interest in AGR-1:

$\mathrm{D}^{\prime}=(500 / \mathrm{d})^{2 *} 10^{[-2.60-(8220 / \mathrm{T})]}$

where

$\mathrm{T}=$ the temperature $(\mathrm{K})$

$\mathrm{d}=$ the fuel kernel diameter in $\mu \mathrm{m}(350 \mu \mathrm{m})$

For the calculations in this work the temperature was assumed to be $1448 \mathrm{~K}$ (1175 C) a temperature within the volume-averaged time-averaged fuel temperature specification.

3.2.2.3 Activity at an FPMS Spectrometer. Given a release rate of $\mathrm{R}$ (atoms/s) from a test capsule into a gas stream flowing at $\mathrm{F}\left(\mathrm{cm}^{3} / \mathrm{s}\right)$ the expected steady state activity $\mathrm{A}_{\mathrm{i}}(\mathrm{Bq})$ in a sample trap of volume $\mathrm{V}\left(\mathrm{cm}^{3}\right)$ viewed by an FPMS detector can be estimated as:

$\mathrm{A}_{\mathrm{i}}=\mathrm{R}_{\mathrm{i}}^{*} \mathrm{~F}^{-1} * \mathrm{~V} * \lambda_{\mathrm{i}}^{*} \exp \left(-\lambda_{\mathrm{i}}^{*} \Delta \mathrm{t}_{\mathrm{t}}\right)$

where
$\mathrm{Ai}=$ the activity in $\mathrm{Bq}\left(\mathrm{Curies}^{*} 3.7 \mathrm{E}+10\right)$ of nuclide $\mathrm{i}$ at the detector
$\mathrm{Ri}=$ the capsule release rate of nuclide $\mathrm{i}$ in atoms $/ \mathrm{s}$
$\mathrm{F}=$ the gas stream flow rate in $\mathrm{cm}^{3} / \mathrm{s}$ (nominal $\left.0.5 \mathrm{~cm}^{3} / \mathrm{s}\right)$
$\mathrm{V}=$ sample trap volume in $\mathrm{cm}^{3}$ (nominal $50 \mathrm{~cm}^{3}$ )
$\lambda \mathrm{I}=$ the decay constant of nuclide $\mathrm{i}$ in $\mathrm{s}^{-1}\left(\lambda=\ln (2) / \mathrm{T}_{1 / 2}\right)$
$\Delta \mathrm{t}=$ transport time from capsule to detector in seconds (nominal $150 \mathrm{~s}$ )

\subsubsection{Spectral Synthesis}

3.2.3.1 Detection Model. The FPMS detectors will be purchased to be similar if not identical to those used in a previous deployment. The sample chamber viewed by each FPMS HPGe detector will be similar if not identical to the "warm traps" used by other similar experiments at the INL.

Detectors in the AGR-1 FPMS will be carefully calibrated to determine their photopeak efficiency as a function of gamma-ray energy. For this work, to predict the performance of those spectrometer the efficiency data from a very similar detector presently implemented at the ATR will be used. This system is designated as warm trap system $\mathrm{W} 1$.

The gamma-ray spectral synthesis program SYNTH does not conveniently accept a user-specified photopeak efficiency curve, but rather it computes response functions based on the specified detector parameters, source and detector geometry, and presence of any absorbers. Since the version of the SYNTH code available for this work ${ }^{\text {xiv }}$ does not allow modeling of collimators, some adjustment of the physical W1 geometry was required. In the actual W1 geometry, the detector views the $4.8 \mathrm{~cm}$ diameter by $3.3 \mathrm{~cm}$ high right circular sample trap through a $3.8 \mathrm{~cm}$ diameter collimator. The source face to detector face distance is $13.4 \mathrm{~cm}$, and source photons pass through about $0.6 \mathrm{~cm}$ of stainless steel before reaching the detector face. This physical geometry was adjusted by repeated trials to develop a SYNTH model that best reproduced the W1 spectrometer response. The accepted model geometry used a $3.3 \mathrm{~cm}$ 
diameter by $3.3 \mathrm{~cm}$ right circular sample volume located $19 \mathrm{~cm}$ from the detector face with $0.2 \mathrm{~cm}$ of iron absorber and a $0.2 \mathrm{~mm}$ thick Ge dead layer at the detector. The sample volume was He-filled at $1 \mathrm{~atm}$.

The model adequacy was tested by specifying to SYNTH a source containing $1,000 \mu \mathrm{Ci}$ $(3.7 \mathrm{E}+7 \mathrm{~Bq})$ each of Eu-152 and Mn-56. This nuclide mix results in spectral peaks from $122 \mathrm{keV}$ to beyond $2600 \mathrm{keV}$. The resultant synthetic spectrum was analyzed using our PCGAP code ${ }^{\text {ix }}$ that routinely analyzes the W1 spectra. The W113410038 efficiency curve used routinely for those analyses was used for this calculation. If the SYNTH model agreed perfectly with the W1 calibration, these analysis results would return $1000 \mu \mathrm{Ci}$ as an activity value calculated from each gamma-ray of interest. The actual results, converted to percentage recovery values (divided by $1000 \mu \mathrm{Ci}$ and converted to percentages), are plotted as a function of gamma-ray energy in Figure 12.

The chosen model gives results within $\pm 5 \%$ of the correct input value over the energy range from about 100 to $2000 \mathrm{keV}$ and within $10 \%$ to $2600 \mathrm{keV}$. This level of accuracy is sufficient for the performance evaluations to follow.

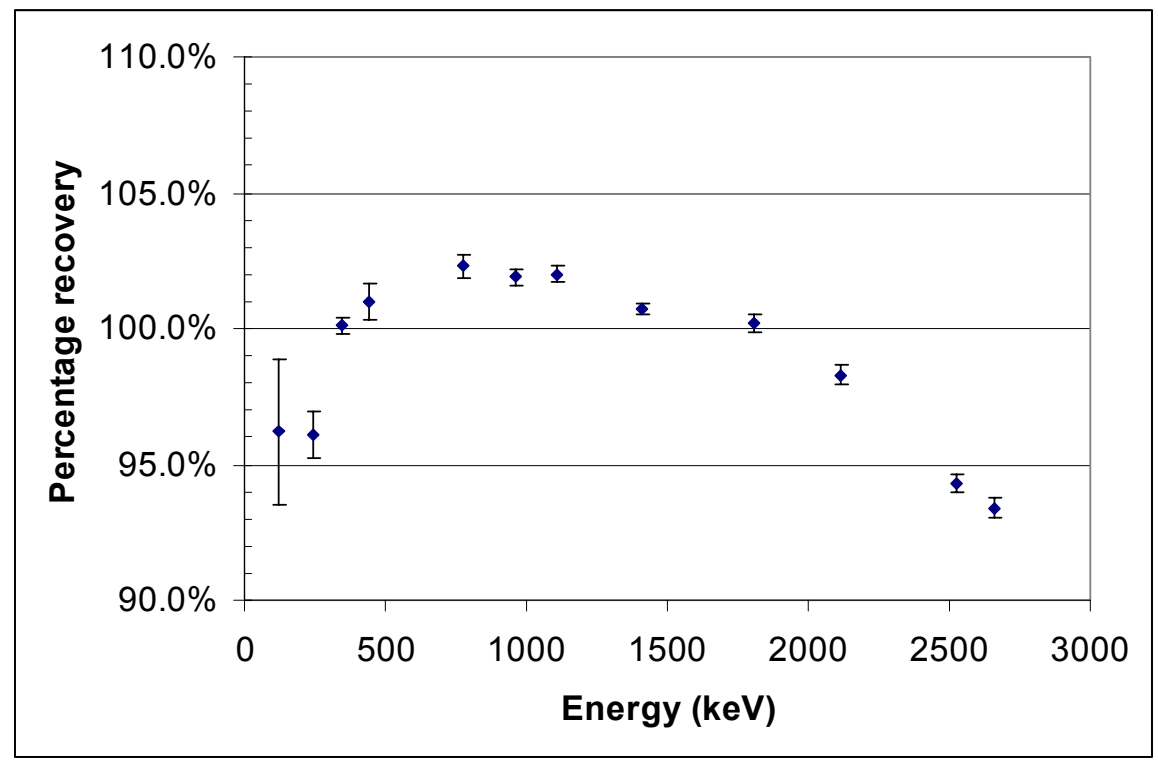

Figure 12. Percentage recovery of input value as a function of gamma-ray energy for the SYNTH model. The plotted error bars are derived from estimated counting statistics only.

3.2.3.2 Synthetic Spectra and Results. Using the sample activities calculated as per Section 3.2.2 and the detection model described in Section 3.2.3.1, a series of synthetic spectra were calculated for a range of AGR-1 conditions. Spectra were calculated from inventory values at 4 effective full power days [EFPD] (near beginning of life), 284 EFPD (near the inventory peak), and at 764 EFPD (beyond the anticipated end of test). Spectra were computed for releases for heavy metal contamination alone (the heavy metal contamination fraction $\left[\mathrm{F}_{\mathrm{HM}}\right]$ was assumed equal to $1.0 \mathrm{E}-04$, the upper $95 \%$ limit of the allowable initial failure fraction), for single particle failures alone, and for the combination of the expected heavy metal release plus the expected release from a single particle failure.

To estimate the effect of fission gas decay products, the nuclide list used for the heavy metal contamination spectra included the short-lived decay products ${ }^{88} \mathrm{Rb},{ }^{89} \mathrm{Rb},{ }^{90} \mathrm{Rb},{ }^{137} \mathrm{Cs},{ }^{138} \mathrm{Cs}$, and ${ }^{139} \mathrm{Cs}$. The decay progeny activities were estimated by assuming that the parent activities predicted for a given irradiation duration flowed through the sample volume for the entire irradiation duration, and that all 
decay products produced plated out in the sample volume. This resulted in a calculational approach analogous to the build up of radioactive species in an initially stable target exposed to a constant neutron flux. Thus:

$$
A_{D}=A_{P}\left(1-e^{-\lambda_{D} T}\right)
$$

where

$$
\begin{aligned}
& \mathrm{A}_{\mathrm{D}}=\quad \text { the estimated daughter activity }(\mathrm{Ci}) \\
& \mathrm{A}_{\mathrm{P}}=\text { the parent activity in the trap at "T" EFPDs }(\mathrm{Ci}) \\
& \lambda_{\mathrm{D}}=\text { the decay constant of the daughter }\left(\mathrm{s}^{-1}\right) \\
& \mathrm{T}=\text { the exposure duration, assumed to be equal to the EFPDs in seconds }
\end{aligned}
$$

For all relevant daughter species other than long lived ${ }^{137} \mathrm{Cs}$, this approach predicts daughter activities in the trap equal to the parent activity. For long-lived ${ }^{137} \mathrm{Cs}$, the exponential "saturation factor" was $1.4 \%$ at 284 EFPD and $4.7 \%$ at 764 EFPD.

These calculations were performed for the nominal flow rate and transport time of $30 \mathrm{~cm}^{3} / \mathrm{minute}$ $\left(0.5 \mathrm{~cm}^{3} / \mathrm{s}\right)$ and 2.5 minutes $(150 \mathrm{~s})$ respectively at irradiation times of 4,284 , and 764 EFPD. The spectral acquisition time was specified to be 10 hours $(36,000 \mathrm{~s})$.

The synthesized spectra were converted to PCGAP format and analyzed relative to a fission gas nuclide library using the efficiency table W113410038 for the W1 warm trap spectrometer. Figure 13 presents an overlay plot of two of the synthesized spectra - the expected background at an irradiation time of 284 EFPDs and the sum of this background and the release from a single particle failure at 284 EFPDs. Figure 14 provides more detail of a spectral portion clearly showing the increase above background for several peaks of interest. Table 1 presents the net fission gas analysis results for the three synthetic composite (single particle failure plus heavy metal background) spectra at 4, 284, and 764 EFPD analyzed with subtraction of the heavy metal background using the concurrent background analysis option of PCGAP. ${ }^{\text {ix }}$ Of particular interest in these results is the excellent precision with which all of the concentrations are determined. When photopeak detection is operating near the detection limit, assay precisions near $\pm 50 \%$. 


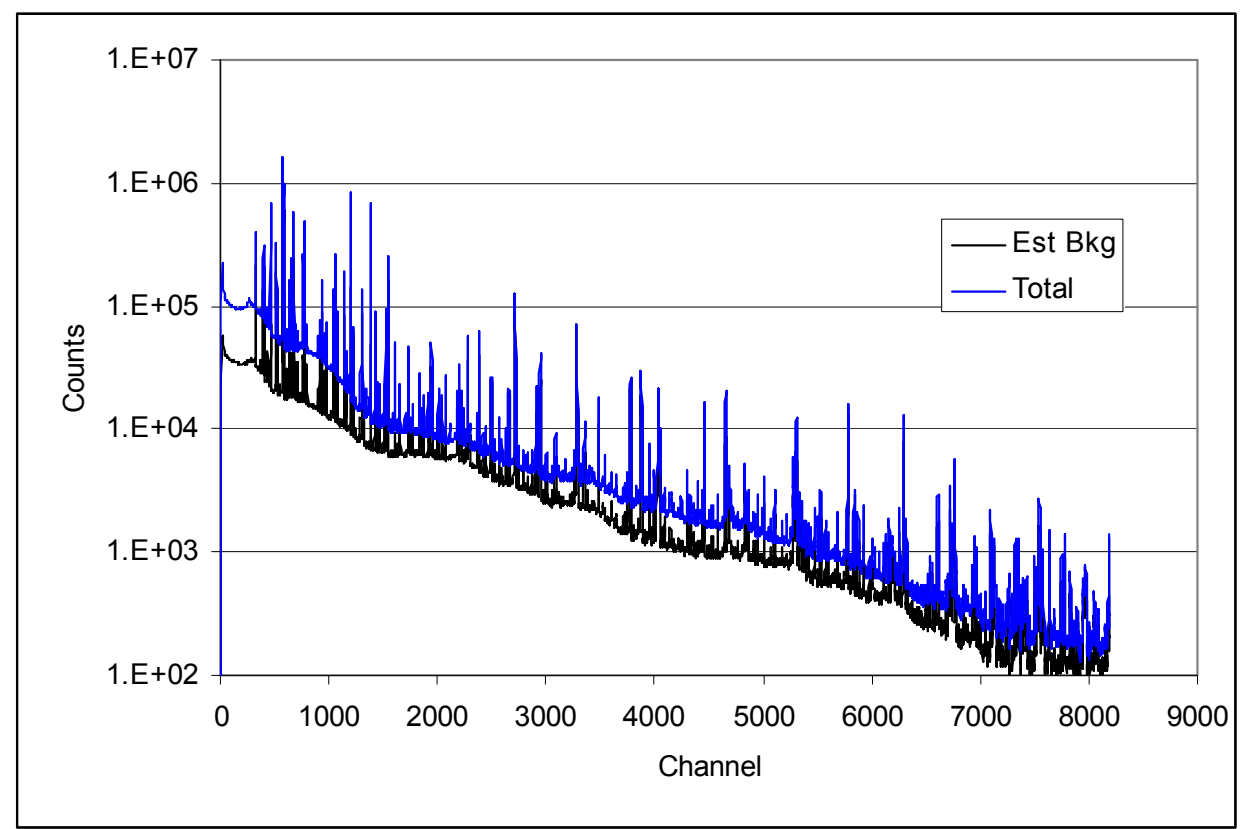

Figure 13. Comparison of the estimated sample + background spectrum resulting from a single particle failure (upper curve) with the expected background from heavy metal contamination (bottom curve). Both synthetic spectra were calculated for 284 EFPDs exposure and a ten hour counting period.

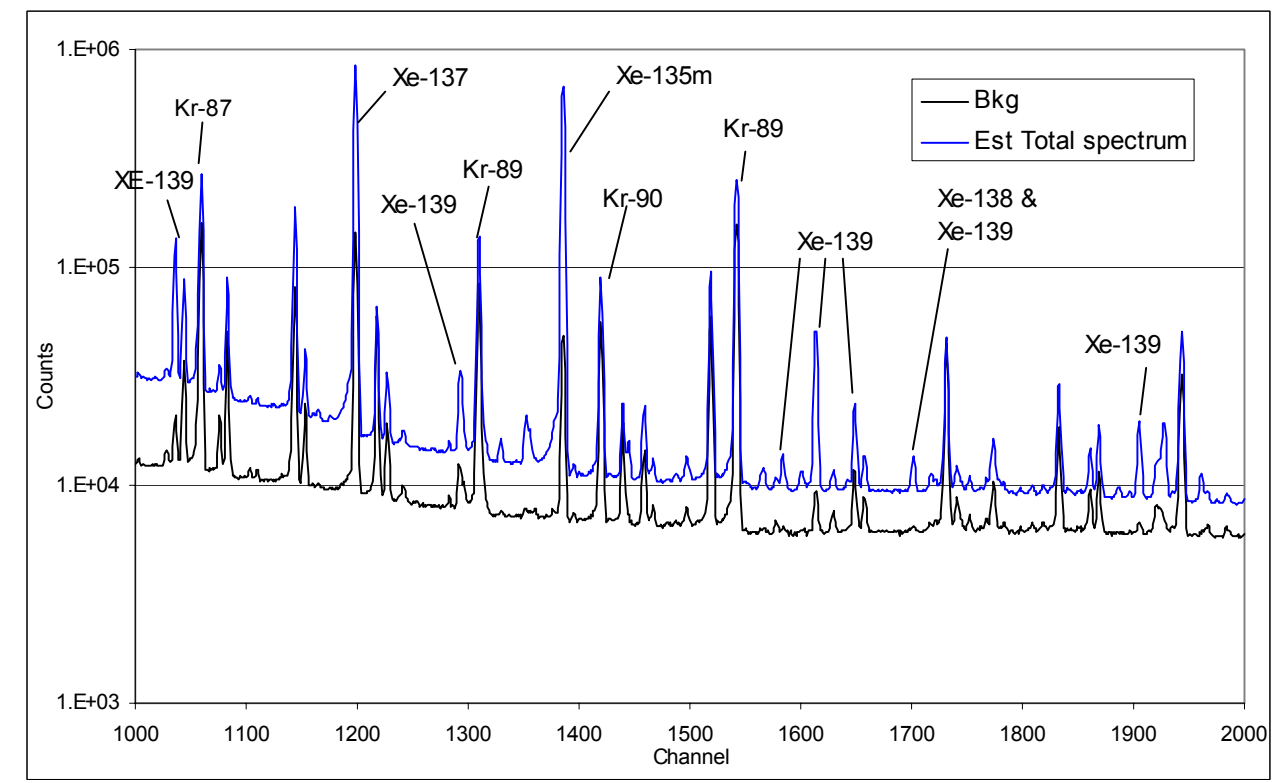

Figure 14. An annotated section (Channels 1000 to 2000) of the spectral comparison of the previous figure. 
Table 1. Synthetic spectra assay results.

\begin{tabular}{|l|l|l|l|c|c|c|c|c|c|}
\hline & \multicolumn{3}{|c|}{4 EFPD } & \multicolumn{3}{c|}{284 EFPD } & \multicolumn{3}{c|}{764 EFPD } \\
\cline { 2 - 11 } Isotope & $\begin{array}{c}\text { Input } \\
\text { Value } \\
(\mu \mathrm{Ci})\end{array}$ & $\begin{array}{c}\text { Analysis } \\
\text { Result } \\
(\mu \mathrm{Ci})\end{array}$ & $\begin{array}{c}\% \\
\text { Error } \\
(1 \sigma)\end{array}$ & $\begin{array}{c}\text { Input } \\
\text { Value } \\
(\mu \mathrm{Ci})\end{array}$ & $\begin{array}{c}\text { Analysis } \\
\text { Result } \\
(\mu \mathrm{Ci})\end{array}$ & $\begin{array}{c}\% \\
\text { Error } \\
(1 \sigma)\end{array}$ & $\begin{array}{c}\text { Input } \\
\text { Value } \\
(\mu \mathrm{Ci})\end{array}$ & $\begin{array}{c}\text { Analysis } \\
\text { Result } \\
(\mu \mathrm{C})\end{array}$ & $\begin{array}{c}\% \\
\text { Error } \\
(1 \sigma)\end{array}$ \\
\hline $\mathrm{Kr}-85 \mathrm{~m}$ & 0.093 & 0.081 & $2.5 \%$ & 0.16 & 0.14 & $2.0 \%$ & 0.033 & 0.029 & $2.4 \%$ \\
\hline $\mathrm{Kr}-87$ & 0.35 & 0.35 & $3.7 \%$ & 0.58 & 0.64 & $4.7 \%$ & 0.12 & 0.13 & $4.3 \%$ \\
\hline $\mathrm{Kr}-88$ & 0.33 & 0.31 & $1.8 \%$ & 0.55 & 0.53 & $1.8 \%$ & 0.11 & 0.11 & $2.8 \%$ \\
\hline $\mathrm{Kr}-89$ & 1.8 & 1.9 & $0.71 \%$ & 3.0 & 3.0 & $0.68 \%$ & 0.57 & 0.58 & $1.1 \%$ \\
\hline $\mathrm{Kr}-90$ & 0.31 & 0.30 & $1.5 \%$ & 0.50 & 0.51 & $0.78 \%$ & 0.095 & 0.092 & $1.6 \%$ \\
\hline $\mathrm{Xe}-135 \mathrm{~m}$ & 1.8 & 1.7 & $11.8 \%$ & 3.6 & 3.3 & $16 \%$ & 1.3 & 1.2 & $11 \%$ \\
\hline $\mathrm{Xe}-137$ & 4.8 & 4.8 & $0.62 \%$ & 8.7 & 8.8 & $0.58 \%$ & 2.7 & 2.8 & $0.67 \%$ \\
\hline $\mathrm{Xe}-138$ & 0.93 & 0.90 & $0.97 \%$ & 1.7 & 1.6 & $0.86 \%$ & 0.48 & 0.47 & $0.86 \%$ \\
\hline $\mathrm{Xe}-139$ & 2.6 & 2.5 & $0.43 \%$ & 4.5 & 4.3 & $0.46 \%$ & 1.2 & 1.2 & $0.45 \%$ \\
\hline
\end{tabular}

\subsubsection{System Sensitivity Estimates}

From the estimated synthetic background spectra (generated from the heavy metal contamination in the test capsules) and the photopeak efficiency of the FPMS spectrometers the detection sensitivity of a typical FPMS spectrometer can be estimated. From Currie, ${ }^{\text {xvi }}$ detection criteria in terms of count rate can be graphically depicted as in Figure 15.

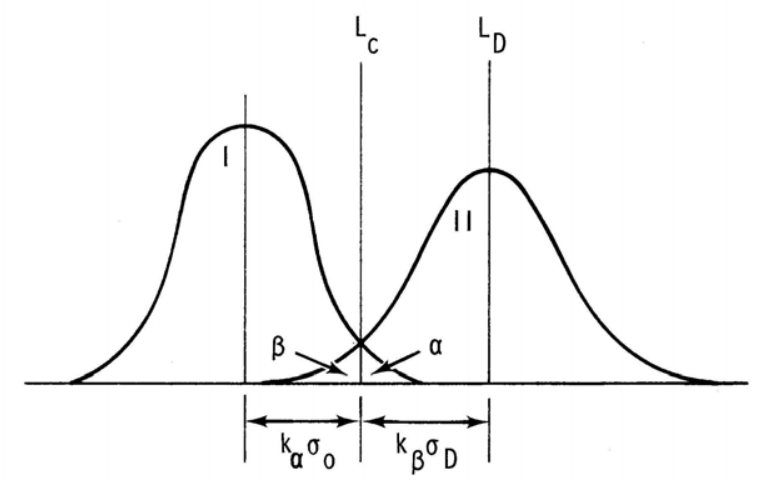

WHERE:

$$
\begin{aligned}
\alpha & =\text { TYPE I ERROR } \\
\beta & =\text { TYPE II ERROR } \\
\sigma_{0} & =\text { STANDARD DEVIATION OF } \hat{R}_{S} \text { WHEN } R_{S}=0 \\
\sigma_{D} & =\text { STANDARD DEVIATION OF } \hat{R}_{S} \text { WHEN } R_{S}=L_{D}
\end{aligned}
$$

Figure 15. Figure depicting the net count rate distributions for the detection sensitivity work. 
Curve I depicts the distribution of net counts expected when the sample counting rate $\left(\mathrm{R}_{\mathrm{S}}\right)$ equals zero. The level $\mathrm{L}_{\mathrm{C}}$ is termed the Decision Level and is the net counting rate that can be assumed different than background with a confidence equal to (100- $\alpha) \%$. For a $5 \%$ significance level (95\% confidence level) $\mathrm{k}_{\alpha}=1.645$. Acquired net count rates on samples can be compared with the decision level to determine if radioactive material is "detected." Analogously, Curve II depicts the distribution of net counts when the mean counting rate equals $\mathrm{L}_{\mathrm{D}}$ (termed by Currie, the "Detection level). Measurements that yield a counting rate equal to $L_{D}$ result in a "detected" decision with $(100-\beta) \%$ confidence. If $\mathrm{k}_{\alpha}=\mathrm{k}_{\beta}=1.645$ then areas $\alpha$ and $\beta$ are $5 \%$ of their respective distribution and the detection rules are operating at about the $95 \%$ confidence interval.

It is important to understand the difference in application of the derived levels $\mathrm{L}_{C}$ and $\mathrm{L}_{\mathrm{D}}$. $\mathrm{L}_{\mathrm{C}}$ is the level that when exceeded by a measured net counting rate indicates that the measured sample has nuclear material that is greater than background (at a 95\% confidence level). $\mathrm{L}_{\mathrm{C}}$ is thus the test level against which measured results are tested to determine if material is "detected." However, it is not the appropriate level to quote if one wishes to specify how much material a measurement process can reliably detect. Since half of a distribution constructed about the $\mathrm{L}_{\mathrm{C}}$ mean is above $\mathrm{L}_{\mathrm{C}}$ ("detected") and half is below ("not detected"), samples containing exactly an $\mathrm{L}_{\mathrm{C}}$ level of material have only a $50 \%$ chance of giving a detected result. If by "reliably" detected one means closer to $95 \%$ detection confidence, a new level must be defined with only $5 \%$ of its distribution below the $\mathrm{L}_{C}$ level. This is the $\mathrm{L}_{\mathrm{D}}$ level.

It can be shown ${ }^{\text {xvii }}$ that, when $\alpha=\beta$ and thus, $\mathrm{k}_{\alpha}=\mathrm{k}_{\beta}$, and, the sample and background counting times are equal $\left(\mathrm{T}_{\mathrm{T}}=\mathrm{T}_{\mathrm{B}}=\mathrm{T}_{\mathrm{C}}\right)$ in terms of counting rate these levels are given by:

$$
\begin{aligned}
& L_{C}=k_{\alpha} \sigma_{0}=k_{\alpha} \sqrt{2} \sigma_{B} \\
& L_{D}=\frac{k_{\alpha}^{2}}{T_{C}}+2 L_{C}
\end{aligned}
$$

where

$$
\begin{aligned}
& \mathrm{k}_{\alpha}=\text { the ordinate of the normal distribution at the desired significance level } \\
& \sigma_{0}=\text { the estimated standard deviation of the null }\left(\mathrm{R}_{\mathrm{S}}=0\right) \text { distribution } \\
& \sigma_{\mathrm{B}}=\text { the estimated standard deviation of the background distribution } \\
& \mathrm{T}_{\mathrm{C}}=\text { the counting time. }
\end{aligned}
$$

In this work when converted to activity units, the level defined by $\mathrm{L}_{\mathrm{D}}$, the amount of material that will be detected with 95\% confidence, will be termed the System Sensitivity Parameter (SSP). Other authors might use the term "minimum detectable activity (MDA)" for this level, but since detected activities are routinely reported for samples that exceed $\mathrm{L}_{\mathrm{C}}$ and are below $\mathrm{L}_{\mathrm{D}}, \mathrm{L}_{\mathrm{D}}$-based sensitivities are clearly not the minimum detectable amount.

The SSP values for the most prominent gamma-ray line of the fission gas nuclides of interest have been calculated for the synthetic gamma-ray spectra from heavy metal contamination releases at an irradiation times of 764 EFPD. The SSPs (in $\mu$ Curies) are compared with the expected release from the failure of a single TRISO particle in Table 2. Since the fission gas releases expected from the failure of a single TRISO particle are much greater than the estimated activity that can be reliably detected (the SSP) these calculations indicate that the FPMS as designed should be capable of detecting the failure of a single particle at any time during the test irradiation. 
Table 2. Calculated system sensitivity parameters compared with the expected release from a single TRISO particle failure at 764 EFPD of irradiation.

\begin{tabular}{lcccc}
\hline Isotope & $\begin{array}{c}\text { Primary } \\
\text { Gamma-ray E } \\
(\mathrm{kev})\end{array}$ & $\begin{array}{c}\text { System Sensitivity } \\
\text { Parameter } \\
(\mu \mathrm{Ci})\end{array}$ & $\begin{array}{c}\text { Single Particle } \\
\text { Release } \\
(\mu \mathrm{Ci})\end{array}$ & $\begin{array}{c}\text { Ratio } \\
\text { Single Particle } \\
\text { Release/SSP }\end{array}$ \\
\hline Kr-85m & 151.2 & 0.0017 & 0.033 & 19 \\
Kr-87 & 402.6 & 0.033 & 0.12 & 3.5 \\
Kr-88 & 2392.1 & 0.009 & 0.11 & 12 \\
Kr-89 & 586 & 0.026 & 0.57 & 22 \\
Xe-135m & 526.6 & 0.003 & 1.3 & 507 \\
Xe-137 & 455.5 & 0.016 & 2.7 & 177 \\
Xe-138 & 258.4 & 0.012 & 0.48 & 39 \\
Xe-139 & 218.6 & 0.011 & 1.2 & 110 \\
\hline
\end{tabular}

\subsection{Discussion}

The on-line gamma-ray spectrometers to be used on the AGR-1 experiment are similar to those of a presently deployed and successful system. Seven spectrometers will be installed, one monitoring each of the six capsule effluent line and one that can monitor any one or combination of effluent gases. Control and data acquisition software will be provided to automate the data acquisition and analysis process. Sensitivity calculations indicate that the planned spectrometers will be able to detect and quantify the fission gases released upon failure of a single TRISO fuel particle. Preliminary calculations reported earlier suggest that the spectrometers will also be able to detect and quantify each particle failure up 20 to 100 successive failures and that the impact of the increased transport times during low flow/high reactor power operation periods is limited to a loss of detection for only the very short lived fission gas nuclides ${ }^{89} \mathrm{Kr}$, ${ }^{90} \mathrm{Kr}$, and ${ }^{139} \mathrm{Xe}^{\mathrm{v}}$. Additional successive particle failures will be detected by the gross radiation monitoring system that is the subject of the next section.

\section{GROSS RADIATION MONITORING SYSTEM}

\subsection{Introduction}

The NaI(Tl) gross detection system that will be part of the AGR FPMS is based on a design that was used successfully during the NPR-1A experiment. ${ }^{\text {ii }}$ The Fission Product Monitoring system will incorporate seven shielded thallium-activated sodium iodide scintillation detectors $[\mathrm{NaI}(\mathrm{Tl})]$ as gross radiation monitors. Each gross radiation monitor will record the relative gamma-ray emission rate of each capsule's effluent as a function of time in order to detect any and every particle failure.

The gross radiation monitor for the AGR-1 configuration incorporates a $\varnothing 25 \mathrm{~mm} \times 25 \mathrm{~mm} \mathrm{NaI(Tl)}$ detector viewing a $25 \mathrm{~mm}$ section of $3 \mathrm{~mm}$ ID effluent line just prior to its entry into the HPGe detector shield. The scintillation detector will be an integral unit provided by the manufacturer with a $38 \mathrm{~mm}$ photomultiplier. ${ }^{\text {xviii }}$ The dividing circuit and preamplifier will be a matching unit from the same manufacturer. ${ }^{\text {xix }}$ The detector will be shielded by a $25-\mathrm{mm}$ thick bismuth shield, and positioned such that it is as close to the viewed sample line segment as feasible. The viewed segment of the effluent line is defined by a specially-fabricated tungsten shield. 
Output pulses from each gross monitor detector will be amplified and then routed to the input of a Canberra Multiport II MCS. A MCS is a multi-channel (in this case 8192 channel) device that acts like a digital strip chart recorder. A dwell time (DT) per channel is set by software control. When data acquisition is started the incoming pulses are "counted" in channel 1 for a length of time equal to the specified dwell time after which the acquisition steps to channel 2 and so on. The result after a time equal to $(8192) *($ dwell time $)$ is a digital strip chart covering the $(8192) *($ dwell time) time period with a count rate of channel contents/dwell time in each channel. The control and analysis software will save the multi-channel scaler spectra showing the $\mathrm{NaI}(\mathrm{Tl})$ gross detector count rate as a function of time. The time range of the spectra will be matched to the sample measurement times used for the corresponding HPGe spectrometer system and the MCS data storage format will be the same as the spectrometer spectra. System control and data readout are via a USB connection to the FPMS control computer.

Detector sensitivity calculations were performed. A transient release model, which mimics the release profile of the NPR-1A experiment, was used to model the gross detector response upon particle failure. A steady state release model was used to predict the background from heavy metal contamination and the steady state releases from any previously failed particles. These scoping calculations and additional experimental results obtained from preliminary laboratory testing of equipment give confidence that the gross monitor detection system will be able to detect each particle failure up to the 250th particle failure. This will satisfy the requirement specified in the AGR-1 test specification. ${ }^{\text {iii }}$ Details of the system design and sensitivity estimates are presented in the following sections.

\subsection{Nal(TI) Gross Detection System Design}

The FPMS consists of seven HPGe spectrometers, and seven gross radiation monitoring systems. The gross radiation monitoring system will incorporate $\mathrm{NaI}(\mathrm{Tl})$ scintillation detectors. The NaI(Tl) detectors (gross monitors) will continuously monitor the associated effluent gas lines for total gamma radiation levels to detect changes in rate. It is important to note that six of the FPMS will be dedicated to individual capsules thus allowing one FPMS the flexibility to measure fission product emission from all six capsules at once or any specific portion of the test train that is of interest.

Figures 16 and 17 show the planned arrangement. Each NaI(Tl) scintillation detector will be shielded with a cast shield of $25 \mathrm{~mm}$ thick bismuth. The sample lines will be shielded with $90 \%$ weight Tungsten (W) with an exposed $25 \mathrm{~mm}$ length viewed by the detector. The detector can be moved up to $10 \mathrm{~cm}$ from the capsule effluent line to reduce the detector counting rate.

A Canberra Multiport II MCS will be used to collect the data obtained from each of the NaI(Tl) detectors. The control and analysis software will save the multi-channel scaler (MCS) spectra showing the $\mathrm{NaI}(\mathrm{Tl})$ detector count rate as a function of time. The time range of the MCS spectra will be matched to the sample measurement times used for the corresponding HPGe spectra.

To assess what equipment would work best with the Canberra Multiport II MCS, two amplifier configurations were tested in order to determine the maximum count rate that could be accurately recorded by the MCS and still maintain reasonable linearity between the input and output (stored) count rate. It is important to understand this behavior in order to determine working count rate limits for the gross monitor. The count rate response of an idealized "point" detector to a point source of a given activity is proportional to the square of the distance between the source and the detector. Measurements were performed with two different amplifiers (and ORTEC 855 and an ORTEC 572) as input to the Multiport II MCS. Amplifier inputs were from a NaI(Tl) detector assembly identical to that planned as an 


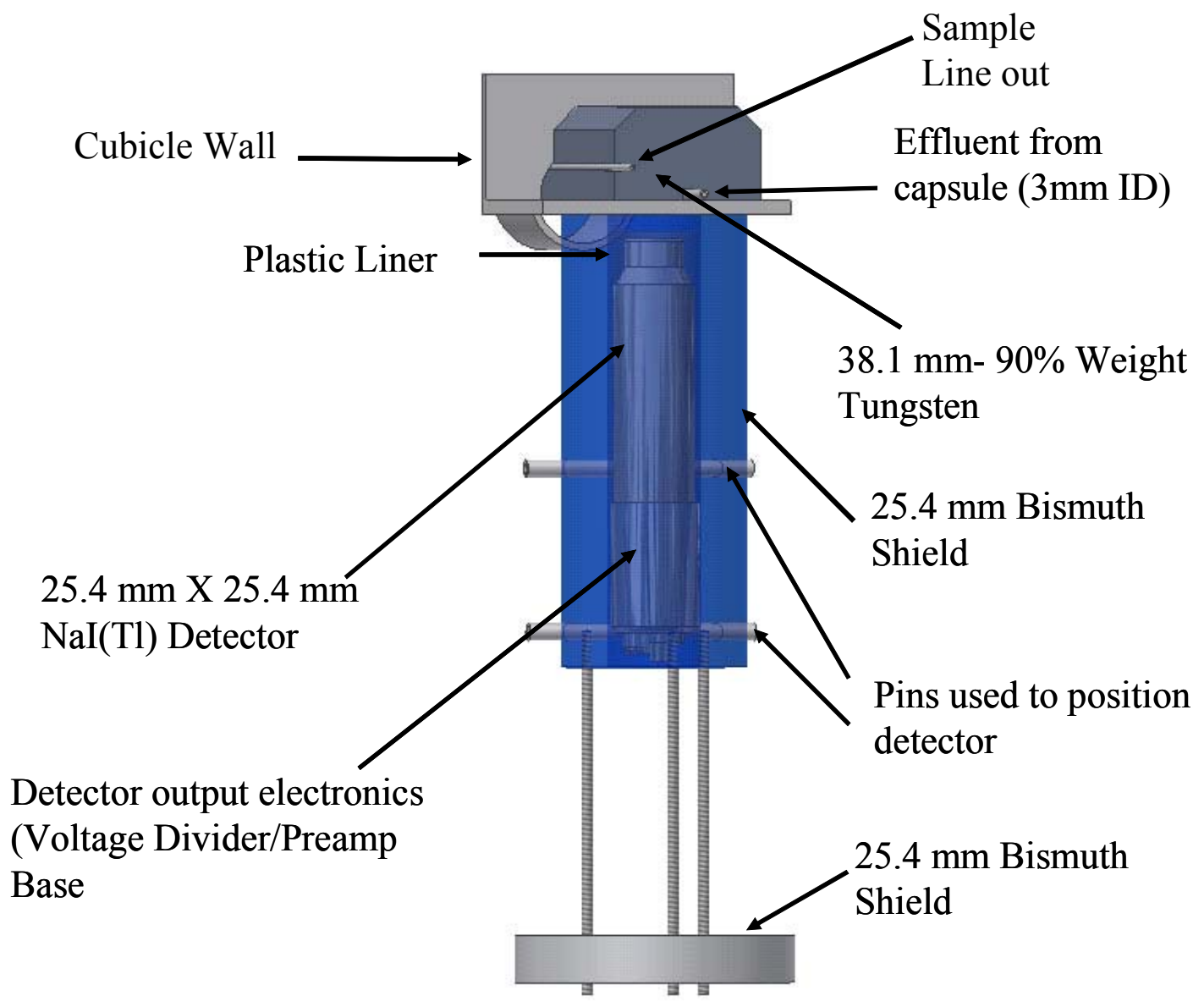

Figure 16. The shield for the NaI(Tl) gross radiation detector will be cast from bismuth. The capsule effluent lines will be shielded with $38 \mathrm{~mm} \mathrm{90 \%} \mathrm{(by} \mathrm{weight)} \mathrm{tungsten.} \mathrm{The} \mathrm{shadow} \mathrm{shield} \mathrm{at} \mathrm{the} \mathrm{base} \mathrm{of}$ the detector will be $25 \mathrm{~mm}$ of bismuth. This detector assembly will be mounted to the cubicle wall between the wall and the spectrometer shield. 

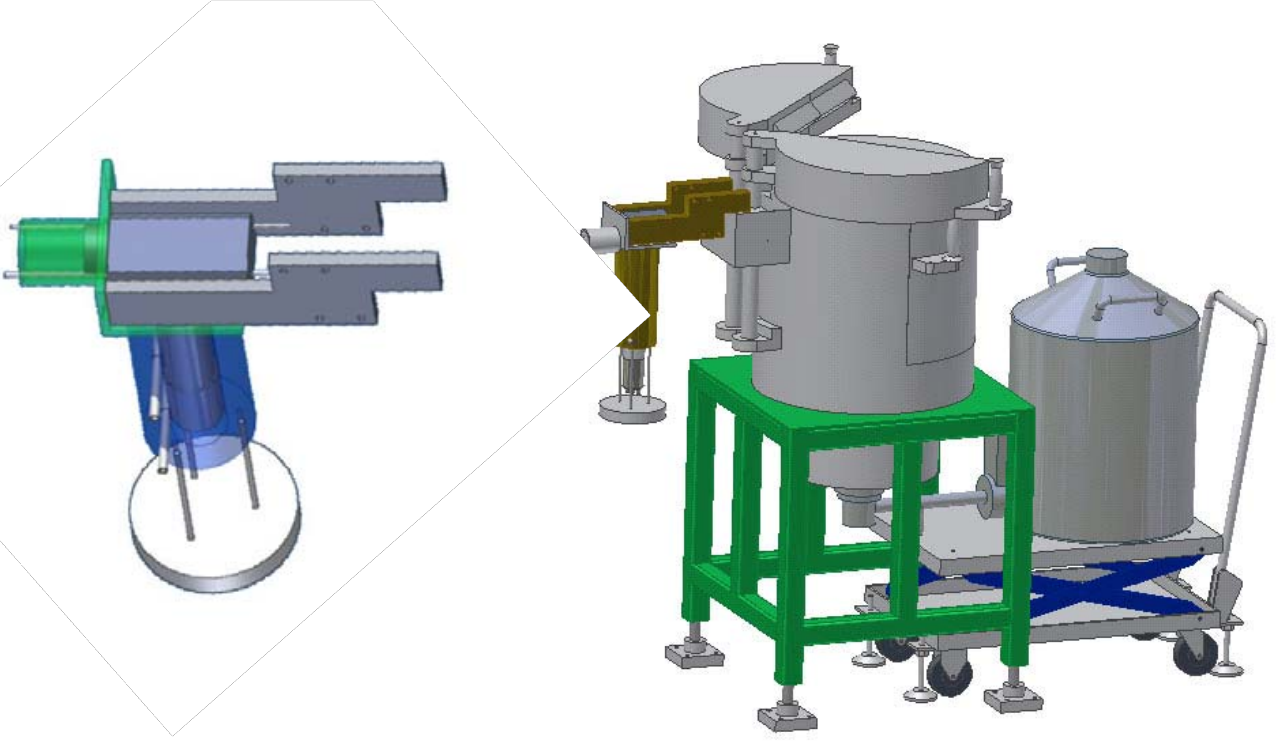

Figure 17. A sketch of one of the FPMS stations showing the gross monitor shield and the HPGe detector and shield.

FPMS gross detector. Figure 18 plots the counting rate recorded in the MCS from a nominally $200 \mu \mathrm{Ci}$ ${ }^{137} \mathrm{Cs}$ source as a function of the square of the source-to-detector distance (corrected for point detector deviations). The ORTEC 572 amplifier was found to have a better linear range than the ORTEC 855 amplifier and thus will be used in the AGR-1 gross detector implementation.

As shown in Figure 18 by using the Model 572 amplifier, the $\mathrm{NaI}(\mathrm{Tl})$ gross detector system remains within $10 \%$ of linear up to about $20,000 \mathrm{cps}$ and is within $20 \%$ of linear at $27,000 \mathrm{c} / \mathrm{s}$. Performance of the less expensive ORTEC 855 amplifier is not as good.

Based on the better linearity of the ORTEC Model 572 amplifier as a function of input rate we have chosen this amplifier for the AGR-1 gross monitoring system. The measured Model 572 performance is equal to or better than that reported for the gross detector system used successfully to count multiple fuel particle failures during NPR-1A. ${ }^{\text {ii }}$ The NPR-1A detector system, in a fashion similar to that designed here, incorporated a feature allowing the detector to be repositioned away from the sample line if count rate dictated. The NPR-1A system "counted" 48 particle failures and never required repositioning.

The following electronic equipment will be used for the AGR-1 gross radiation monitoring system:

- $\quad 25 \mathrm{~mm} \times 25 \mathrm{~mm}$ Bicron $\mathrm{NaI}(\mathrm{Tl})$ detector with a PA-12 voltage divider/preamp base

- $\quad$ ORTEC -572 spectroscopy amplifier

- $\quad$ ORTEC -556 HV power supply

- $\quad$ Canberra Multiport II MCS.

Each Multiport II services four inputs, thus two will be required for the seven inputs. 


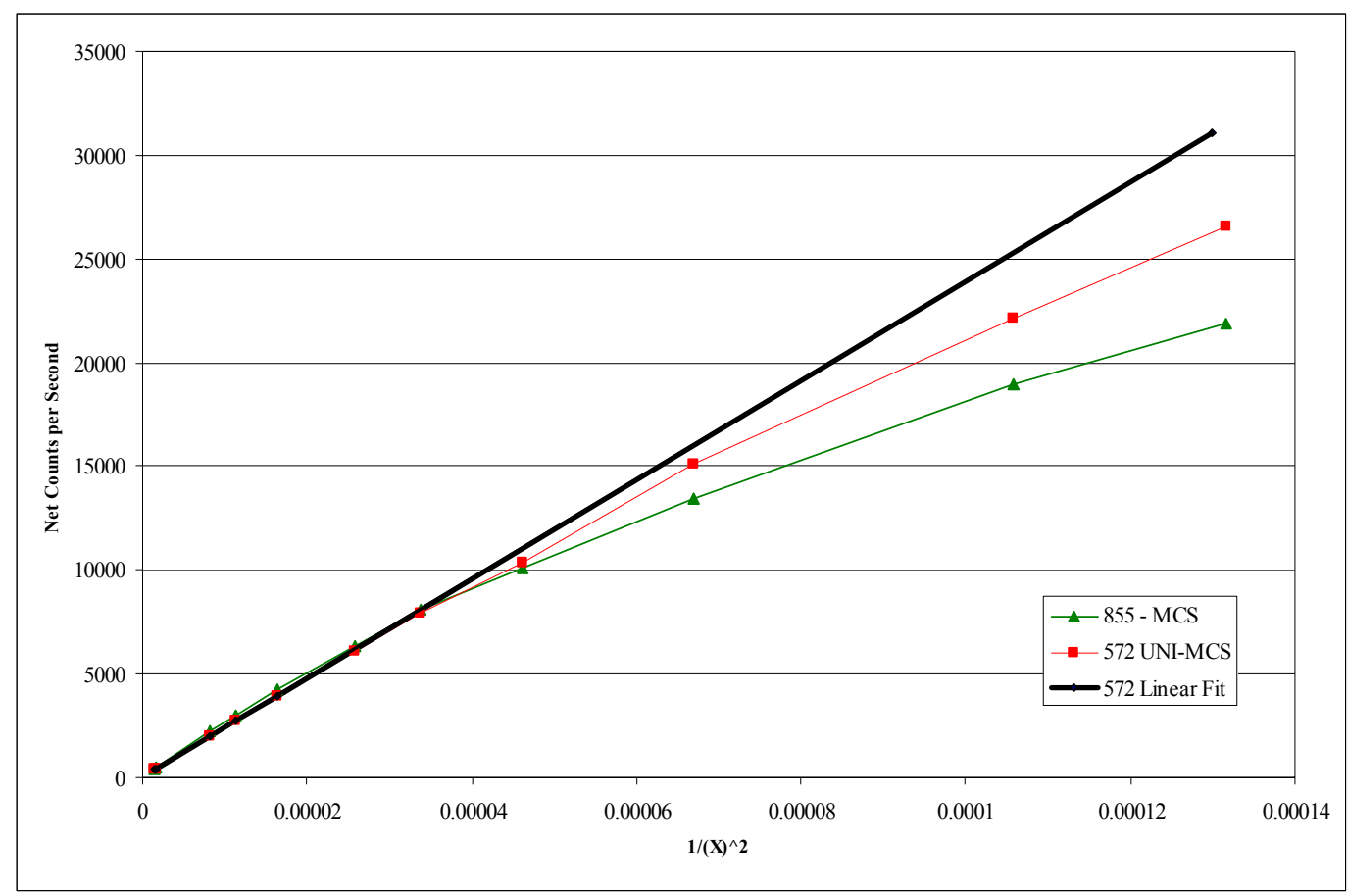

Figure 18. Net counts per second versus $1 / \mathrm{x}^{2}$ for two amplifier-to-Mutiport II configurations. The ORTEC 572 exhibits better count rate linearity than the ORTEC 855.

\subsection{Gross Monitor Sensitivity Calculations}

Calculations were performed to estimate the activity at the face of the $\mathrm{NaI}(\mathrm{Tl})$ gross detector for different release scenarios and to determine if this detector could detect each fuel particle failure up to and including the 250th failure. The calculations used a transient release model modeled after the gross detector response noted in NPR-1A for a particle failure. A scanned image of the NPR-1A gross detector response to the first particle failure is presented in Figure 19. The X-axis of this plot is in MCS channel number (covert to time in seconds using 5.3 seconds/channel) while the $\mathrm{Y}$-axis is counts per channel. The spectrum covers a 24 hour time period.

Note that prior to the particle failure the gross count was quite low (about $25 \mathrm{c} / \mathrm{s}$ ), as the activity from the particle release reached the detector the count rate spiked to about $13,000 \mathrm{c} / \mathrm{s}$ and then recovered to about $140 \mathrm{c} / \mathrm{s}$. The spike had a duration of about 100 seconds. We have developed a model for the AGR-1 transient releases that closely mimics this response.

The response model assumes that the gross detector counting rate is directly proportional to the total fission gas activity in the viewed segment of the effluent line at the gross detector location. The viewed activity prior to any particle failure is presumed to be that predicted for steady state releases from the heavy metal contamination in the monitored capsule while the steady state activity level after the particle failure transient is the sum of that from heavy metal contamination and from the failure of a single particle (see Section 3.2). Similarly after 249 particle failures the background above which the 250 th release must be detected is the sum of the steady state release from 249 particles and the heavy metal contamination. The transient release model is developed as follows. 


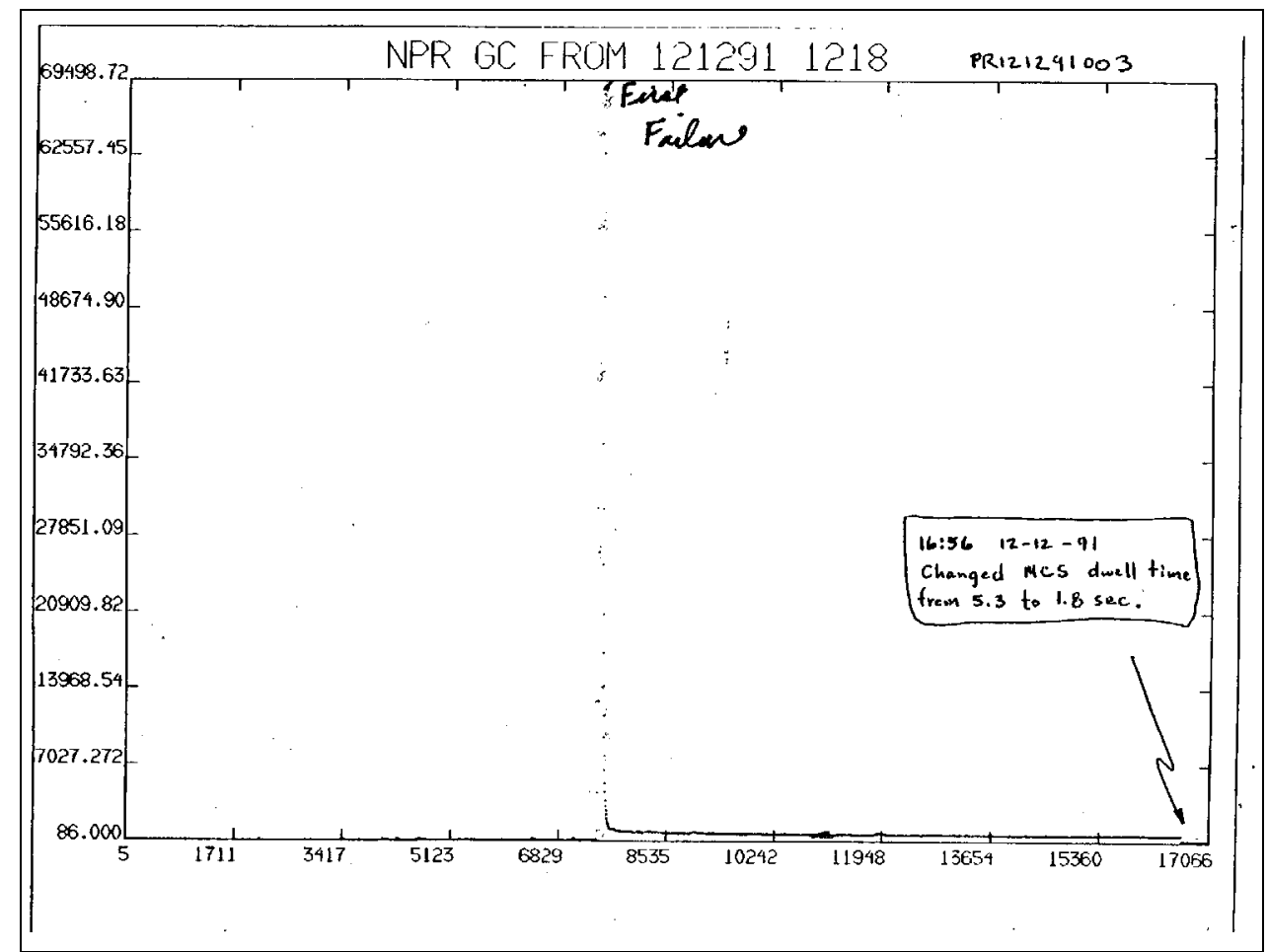

Figure 19. Scanned image of the gross monitor response to the first particle failure from the NPR-1 Test. The X-axis is MCS channels (5.3 s/channel) while the Y-axis represents the number of counts. From the data shown in this plot the spike maximum is $13,113 \mathrm{cps}$. PR121241003 is the corresponding gamma-ray information obtained for this run.

We define the released fraction $\left(\mathrm{f}_{\mathrm{i}}\right)$ as the ratio of the atoms of nuclide i released upon particle failure $\left(\mathrm{I}_{i R}\right)$ to the total atoms of $\mathrm{i}$ in the particle inventory $\left(\mathrm{I}_{i P}\right)$. Although not exactly equivalent, the best estimate that we have for $f_{i}$ is the release rate-to-birth rate ratio of $i\left(R_{i} / B_{i}\right)$. This is defensible both in the limit for very short-lived nuclides where the total inventory closely follows the birth rate and in the limit of very long-lived or stable nuclides where the release fraction can be formulated as:

$$
f_{i}=\frac{R_{i} * t}{B_{i} * t}
$$

where $t$ is the irradiation duration, $\mathrm{R}_{\mathrm{i}}$ and $\mathrm{B}_{\mathrm{i}}$ are the release and birth rates respectively.

Then by definition we can write: 
$f_{i}=\frac{I_{i R}}{I_{i P}}$

rearranging and substituting gives :

$I_{i R}=I_{i P} * f_{i} \cong I_{i P} *\left(R_{i} / B_{i}\right)$

presuming the release occurs in a time $t_{R}$ then the release rate $R_{i}$ in atoms/s

during a transient of duration $t_{r}$ seconds is simply

$R_{i}=\frac{I_{i R}}{t_{r}} \approx \frac{I_{i P} *\left(R_{i} / B_{i}\right)}{t_{r}}$ and as an activity release rate $\left(A_{i}\right)$ in $\mathrm{Bq} / \mathrm{s}$ is

$A_{i}=R_{i} * \lambda_{i} \approx \frac{I_{i P} *\left(R_{i} / B_{i}\right) * \lambda_{i}}{t_{r}}$

at a nominal gas flow rate of $F \mathrm{~cm}^{3} / \mathrm{s}$ and a capsule - to - detector transport time of $t_{T}$ seconds, the activity concentration $\left(C_{i}\right)$ at the gross detector during the transient duration can be estimated as :

$C_{i}=\frac{A_{i} * e^{-\lambda_{i} * t_{T}}}{F} \approx \frac{I_{i P} *\left(R_{i} / B_{i}\right) * \lambda_{i} * e^{-\lambda_{i} * t_{T}}}{t_{r} * F}$

It is interesting to contrast this transient release with the formulation for the steady state release from a single particle failure. The calculation of Section 3.2.2.3 for the steady state released concentration from a failed particle can be formulated as:

$C_{i}^{\prime}=\frac{B_{i} *\left(R_{i} / B_{i}\right) * \lambda_{i} * e^{-\lambda_{i} * t_{T}}}{F}$

where the birth rate $B_{i}$ is assumed equal to the inventory decay rate. Thus:

$C_{i}^{\prime}=\frac{I_{i P} * \lambda_{i} *\left(R_{i} / B_{i}\right) * \lambda_{i} * e^{-\lambda_{i} * t_{T}}}{F}$

then

$\frac{C_{i}}{C_{i}^{\prime}} \cong \frac{1}{\lambda_{i} * t_{r}}=\frac{t_{1 / 2}}{\ln 2 * t_{r}}$

a result that indicates that the magnitude of the transient release concentration relative to the steady state release is directly proportional to the half life of the species of interest and inversely proportional to the transient release duration. This is an intuitive result.

Since the gross detector system responds to the total gamma-ray activity in the viewed section of the sample line the actual response (D) in c/s can be expressed as: 
$D=K * \sum_{i}\left(k_{i} * C_{i}\right)$

where $K$ is a calibration factor dependent on the detector and source geometry and $k_{i}$ is an isotope-specific constant dependent on the gamma-ray energies and yields. Consequently the detector response for any given geometry and isotopic mix is proportional to the sum of the isotopic concentrations of the contributing nuclides in the viewed segment of the sample line.

The transient release model has been applied to the AGR-1 experiment. The particle inventories were from the MCWO2 inventory. ${ }^{\mathrm{xi}}$ The release rate-to-birth rate ratios were those computed in Section 3.2.2.

For example, consider $\mathrm{Kr}^{85 \mathrm{~m}}$ at 4 EFPD. In this case, the capsule inventory (Node 69, stack 3 ) is $0.654 \mathrm{Ci} / \mathrm{cc}$, the decay constant $\lambda$ is $4.30 \times 10^{-5} \mathrm{~s}^{-1}$, and the calculated release-to-birth ratio for a failed fuel particle is 0.046 . If the transient release duration $\left(t_{r}\right)$ is assumed to be 60 seconds, the activity per $\mathrm{cm}^{3}$ at the gross monitor during the transient release is estimated to be:

$\mathrm{C}_{85 \mathrm{~m}}=2.66 \mathrm{E}+04 \mathrm{~Bq} / \mathrm{cm}^{3}=7.2 \mathrm{E}-07 \mathrm{Ci} / \mathrm{cm}^{3}$

The formulation of Section 3.2.2.3 estimates the steady state releases from heavy metal contamination prior to any particle failure to be:

$$
C_{85 m}^{\prime}(H M)=3.1 E-09 \mathrm{Ci} / \mathrm{cm}^{3}
$$

and the steady state release rate of ${ }^{85 \mathrm{~m}} \mathrm{Kr}$ from a failed fuel particle to be:

$$
C_{85 m}^{\prime}(F F)=1.85 E-09 \mathrm{Ci} / \mathrm{cm}^{3} \text {. }
$$

Thus our model suggests that at 4 EFPD of irradiation and prior to any particle failure the gross detector system is viewing a ${ }^{85 \mathrm{~m}} \mathrm{Kr}$ activity of around $3.1 \mathrm{E}-09 \mathrm{Ci} / \mathrm{cm}^{3}$. If a particle were then to fail, the ${ }^{85 \mathrm{~m}} \mathrm{Kr}$ activity would "spike" to about $7.2 \mathrm{E}-07 \mathrm{Ci} / \mathrm{cm}^{3}$ for about a minute and then recover to a concentration of about $5.0 \mathrm{E}-09 \mathrm{Ci} / \mathrm{cm}^{3}$. This spike of more than 2 orders of magnitude will be easily detectable.

Figure 20 presents the simulated response of the gross detector to the summed estimated fission gas releases before, during, and after a single particle failure. The inventory is that at 244 EFPD and the transient release duration is assumed to be 60 seconds. Note the similarity in shape between this plot and the plot from the NPR-1A spectrometer during the first particle failure (Figure 19). It is important to note that these are simulated responses, and actual results, particularly after multiple particle failures, are significantly more complicated. During the NPR-1A experiment ONLY the gross detector data from the first particle failure was as "clean" and indicative as the plot of Figure 19. All of the following traces had several peaks large and small some due to additional particle failures and some due to the kinetics of releases from earlier failed particles. The simulated traces are simply to show that the planned system has the sensitivity to perform adequately, not to reproduce any expected actual trace. 


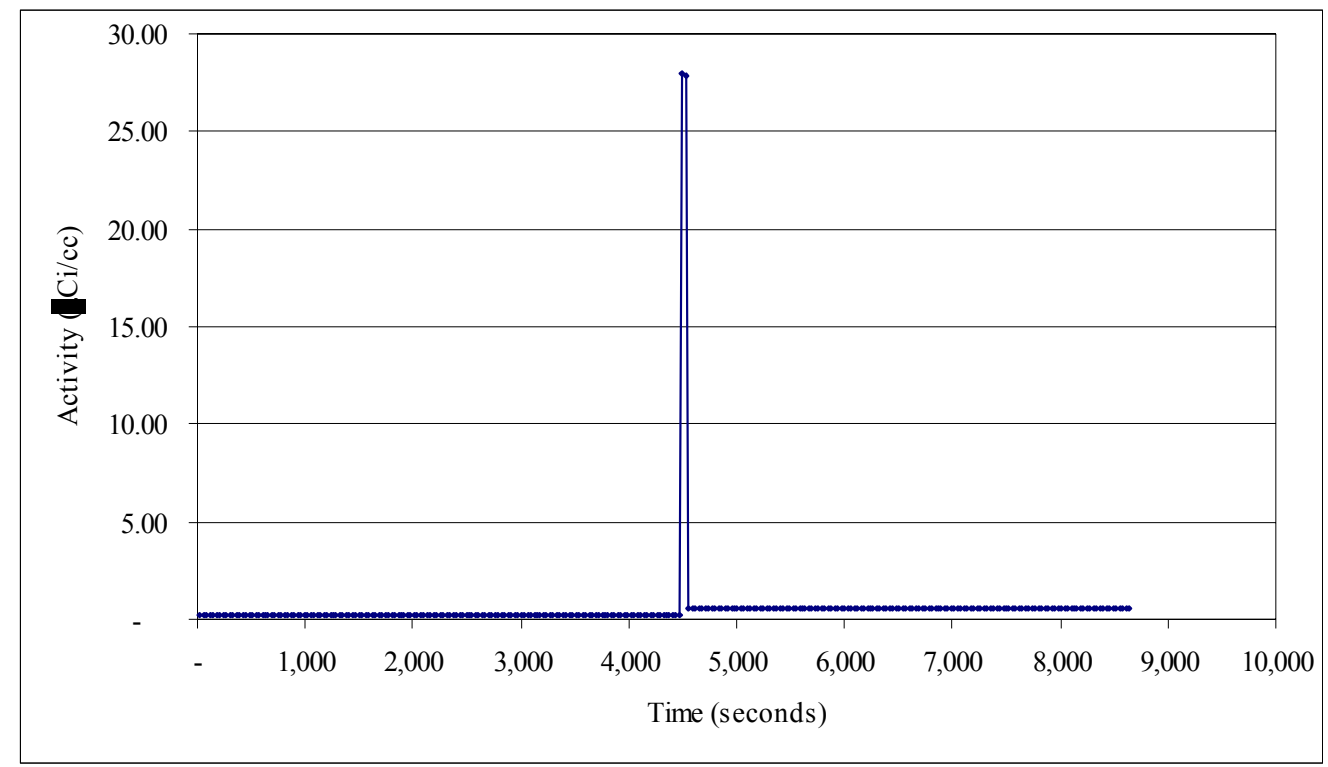

Figure 20. The simulated response of the AGR-1 gross detector system to the failure of a single particle at 244 EFPD.

A similar calculation was performed assuming 250 prior particle failures and simulating the detector response to the 251st failure. That result is presented in Figure 21.

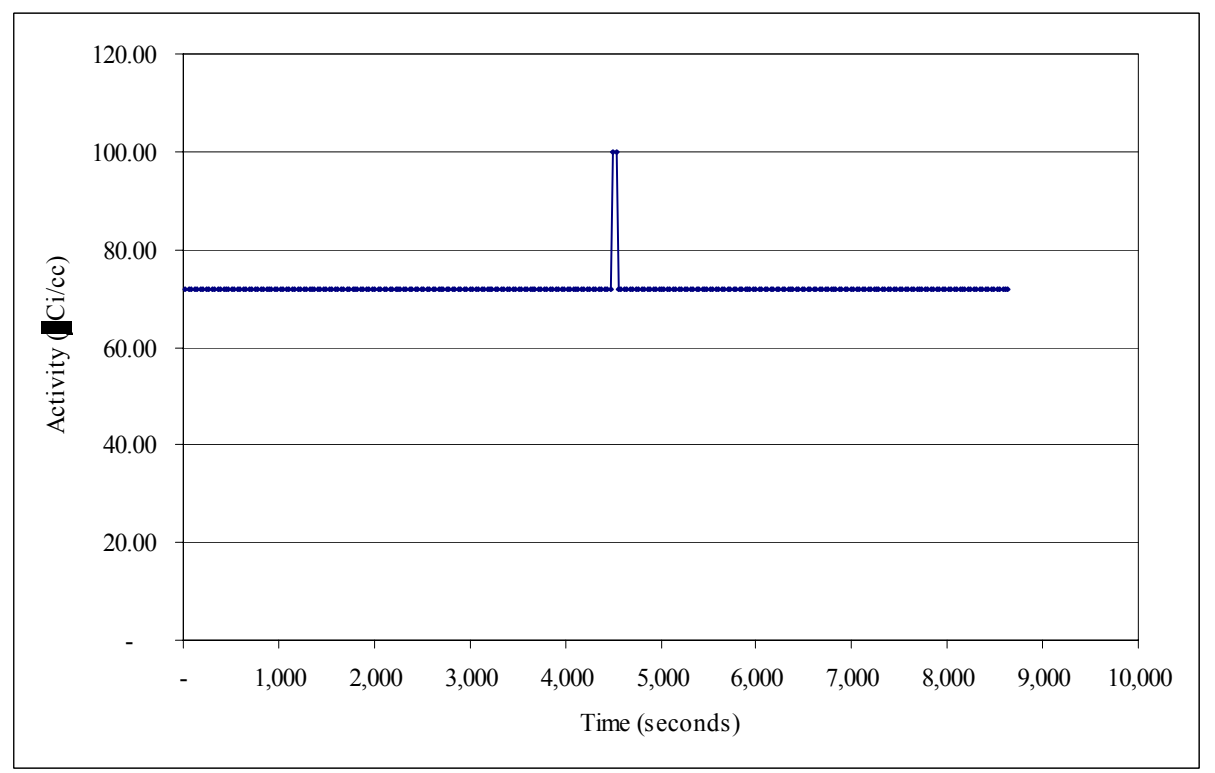

Figure 21. The simulated response of the AGR-1 gross detector system to the failure of the 251st particle at 244 EFPD. 
Similar calculations have been performed to predict release concentrations throughout the test duration. These results are summarized in Figure 22. Curves depict the estimated peak release concentration from a single particle failure (bottom curve), the estimated steady-state release concentration form 250 prior particle failures (middle curve) and the estimated total release from the 251st failure. Note that on day 244 EFPD the estimated maximum activity during one particle failure is $28 \mu \mathrm{Ci} / \mathrm{cm}^{3}$ and after the 250 th particle failure the steady state activity is $72 \mu \mathrm{Ci} / \mathrm{cm}^{3}$, and at the end of life the particle release and "background" values are 8.1 and $19 \mu \mathrm{Ci} / \mathrm{cm}^{3}$ respectively. Rough detectability estimates $^{\mathrm{xvii}}$ presuming the concentration-to-count rate conversion derived from the NPR-1A gross detector (about $70 \mathrm{c} / \mathrm{s} / \mu \mathrm{Ci} / \mathrm{cm}^{3}$ of fission gas activity) and 1 second dwell times suggest that at 244 and 764 EFPD concentration increases of about $5 \mu \mathrm{Ci} / \mathrm{cm}^{3}$ and $3 \mu \mathrm{Ci} / \mathrm{cm}^{3}$ respectively should be detectable with $95 \%$ confidence. It seems clear from these estimates that the gross detection system designed for the AGR-1 experiment is sufficiently sensitive to meet the programmatic requirement of detecting each particle failure up to and including the 250th.

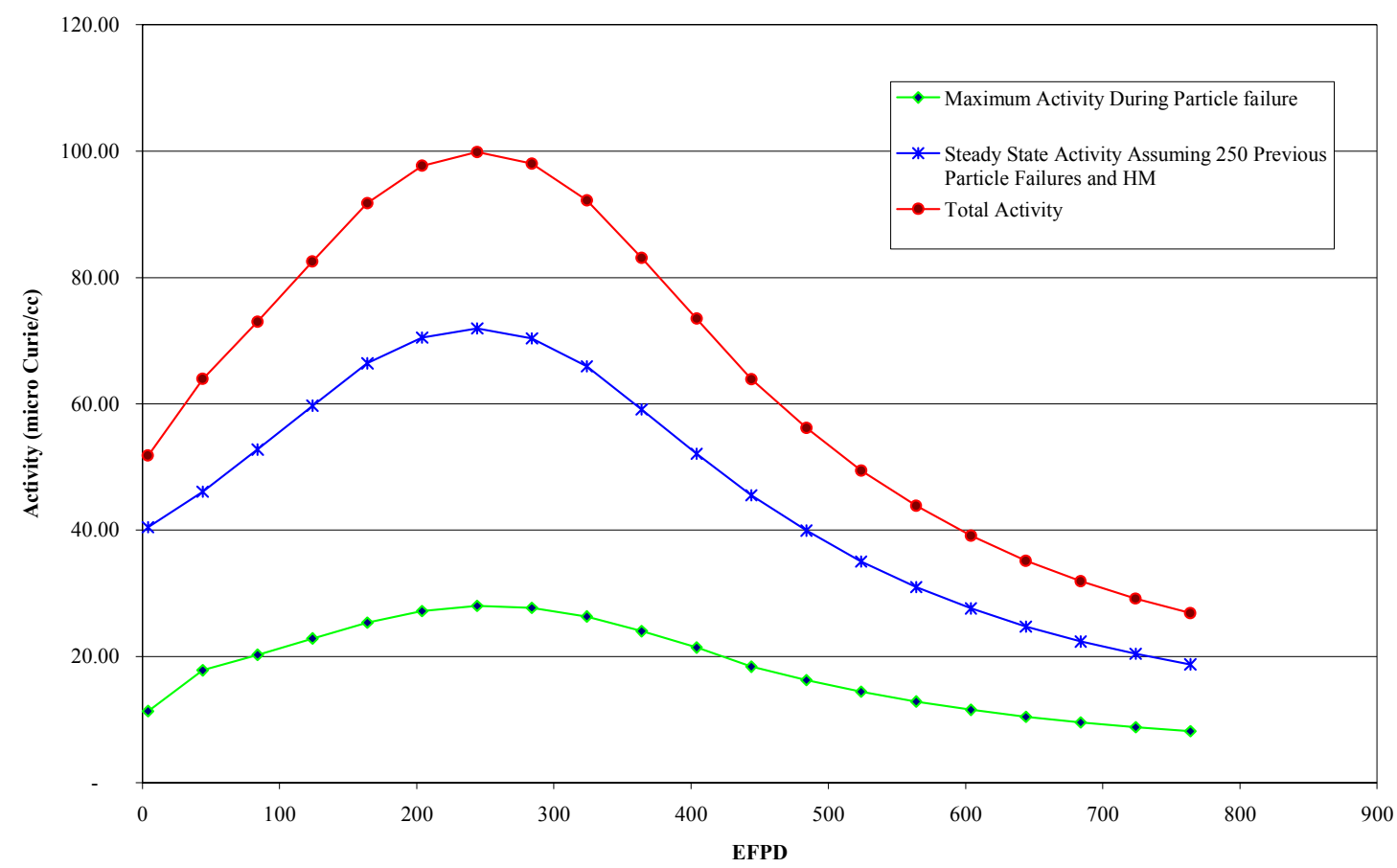

Figure 22. Estimated total activity per $\mathrm{cm}^{3}$ at a given gross detector as a function of test duration. Curves depict the estimated release concentration from a single particle failure (bottom curve), the estimated steady-state release concentration form 250 prior particle failures (middle curve) and the estimated total release from the 251 st failure (top curve).

It is interesting to correlate the gross detector response noted during the first particle failure during the NPR-1A experiment with the corresponding spectrometer results. The NPR-1A experiment used the same fission product detection operating philosophy that we plan to use in the AGR experiment series; namely, the start and stop times of the gross detector and spectrometer measurements were coordinated, thus there is a gamma-ray spectrum (ID PR121241003) that covers the time period of the gross detector plot of Figure 19. The total isotopic fission gas concentrations measured in the 24 hour spectrum before PR121241003 started was $9.4 \mathrm{E}-04 \mu \mathrm{Ci} / \mathrm{cm}^{3}$. Spectrum PR121241003 recorded a total fission gas concentration of $0.15 \mu \mathrm{Ci} / \mathrm{cm}^{3}$. We have estimated that $94 \%$ of the activity measured during the 24 hour 
count time of spectrum PR121241003 was released during the transient peak that had a duration of about 90 seconds. This suggests a total fission gas concentration of about $135 \mu \mathrm{Ci} / \mathrm{cm}^{3}$ during the transient release. This value is reasonably comparable with our estimate of around $100 \mu \mathrm{Ci} / \mathrm{cm}^{3}$ predicted for a single particle failure in the AGR-1 experiment at 244 EFPD. A direct comparison between the tests is not reasonable since the irradiation histories and fuel loadings for the NPR-1A experiment are different than those for AGR-1; however, since the gross monitor configurations are comparable between the two experiments the concentration comparison gives confidence that the AGR-1 gross detector will perform much like that of the NPR-1A experiment. During the NPR-1A experiment approximately 48 particle failures were observed by the NPR-1A gross monitoring system, and there is no reason to believe that it could not have "counted" several more.

\subsection{Summary}

The NaI(Tl) gross monitor system designed for the AGR-1 experiment was based on the successful NPR-1A gross monitor. The AGR-1 experiment has six monitors viewing six different capsule effluent lines. One additional monitor will have the ability to measure fission product emission from all six capsules at once or any one effluent line that is of interest.

Each $\mathrm{NaI}(\mathrm{Tl})$ monitor will be individually shielded and will have the flexibility to be moved from the effluent line to reduce gamma-ray fluence at the face of the detector as needed. A Canberra Multiport II MCS will be used to collect the data obtained from each of the NaI(Tl) detectors. The supporting electronics have been chosen to maximized the response linearity of the monitoring system. The control and analysis software will save the multi-channel scaler spectra showing the NaI(Tl) detector count rate as a function of time. The time range of the spectra will be matched to the sample measurement times used for the corresponding HPGe spectra.

A release model was developed to model the transient releases during particle failures. These calculations indicate the gross monitor system should easily meet the programmatic requirement of detecting each particle failure up to and including the 250th.

\section{REFERENCES}

i. J. K. Hartwell and E. W. Killian, "On-line Gamma-Ray Data Acquisition at the Power Burst Facility,” Nuc. Instr. and Methods in Phys. Research, A242, p. 487, January 1986.

ii. C. V. McIsaac et al, "Concentrations of Fission Product Noble Gases Released During the NP-MHTGR Fuel Compact Experiment-1A”, ST-PHY-92-032, April 1992.

iii. J. T. Maki, “AGR-1 Irradiation Test Specification” EDF-4731, July 2004.

iv. INL Drawing number 628627, “ATR AGR System 1 Flow Diagram,” 2005.

v. J. K. Hartwell, "An Assessment of the Detection Sensitivity of the AGR-1 Fission Product Spectrometers”, EDF-5134, 09-AUG-2004.

vi. $\quad$ Canberra, Inc., "Model 767S Shield”, Drawing B202454, Feb. 2000.

vii. J. K. Hartwell, S. G. Goodwin, L. O. Johnson, E. W. Killian, R. L. Kynaston, and R. L. Heath, "Pulse Injection with Subsequent Removal for Gamma-Ray Spectrometry," US 4968889, Granted November 1990. 
viii. Canberra Ind., "Outline Drawing Cryostat Model 7915-30/S RDC-2," Canberra Drawing B201143X, October 2000.

ix. E. W. Killian and J. K. Hartwell, "PCGAP: Users Guide and Algorithm Description," INEEL/EXT-2000-00908, Sept. 2000.

x. J. T. Maki, “AGR-1 Irradiation Experiment Test Plan,” INL/EXT-05-00593, August 2005.

xi. G. S. Chang, "MCWO - Linking MCNP AND ORIGEN2 for Fuel Burnup Analysis," To be published in the Proceedings of "The Monte Carlo Method: Versatility Unbounded In A Dynamic Computing World,' Chattanooga, Tennessee, April 17-21, 2005, on CD-ROM, American Nuclear Society, LaGrange Park, IL (2005)

xii. G. S. CHANG, "Final Neutronics Evaluations for the AGR-1 Experiment in ATR East Position B-10," Idaho National Laboratory, EDF-5683 (DRAFT), March, 2005.

xiii. INEEL, "Development of Improved Models and Designs for Coated-Particle Gas Reactor Fuels: Annual Progress Report under the International Nuclear Energy Research Initiative (I-NERI)," INEEL/EXT-03-01349, November 2003.

xiv. W. K. Hensley et al, "SYNTH for Windows, Version 4.11," Pacific Northwest Laboratory, Richland, WA (2000). Also W. K. Hensley, E. A. Lepel, "SYNTH: A New Tool For Estimating Interferences In Instrumental Neutron Activation Analysis", J. Radioanal. Nucl. Chem., 233, 1998.

xv. G. Chang, Jack-2 AGR-1 Inventory, 1/29/05.

xvi. L. A. Currie, "Limits for qualitative detection and quantitative determination," Anal. Chem. 40, p 586, 1968.

xvii. J. K. Hartwell, "Detection Limits for Radioanalytical Counting Techniques," ARH-SA-215, June 1975

xviii. Bicron Model 1M1/1.5

xix. Bicron Model PA-12 


\section{Appendix A}

\section{Spectrometer Assembly Photographs}




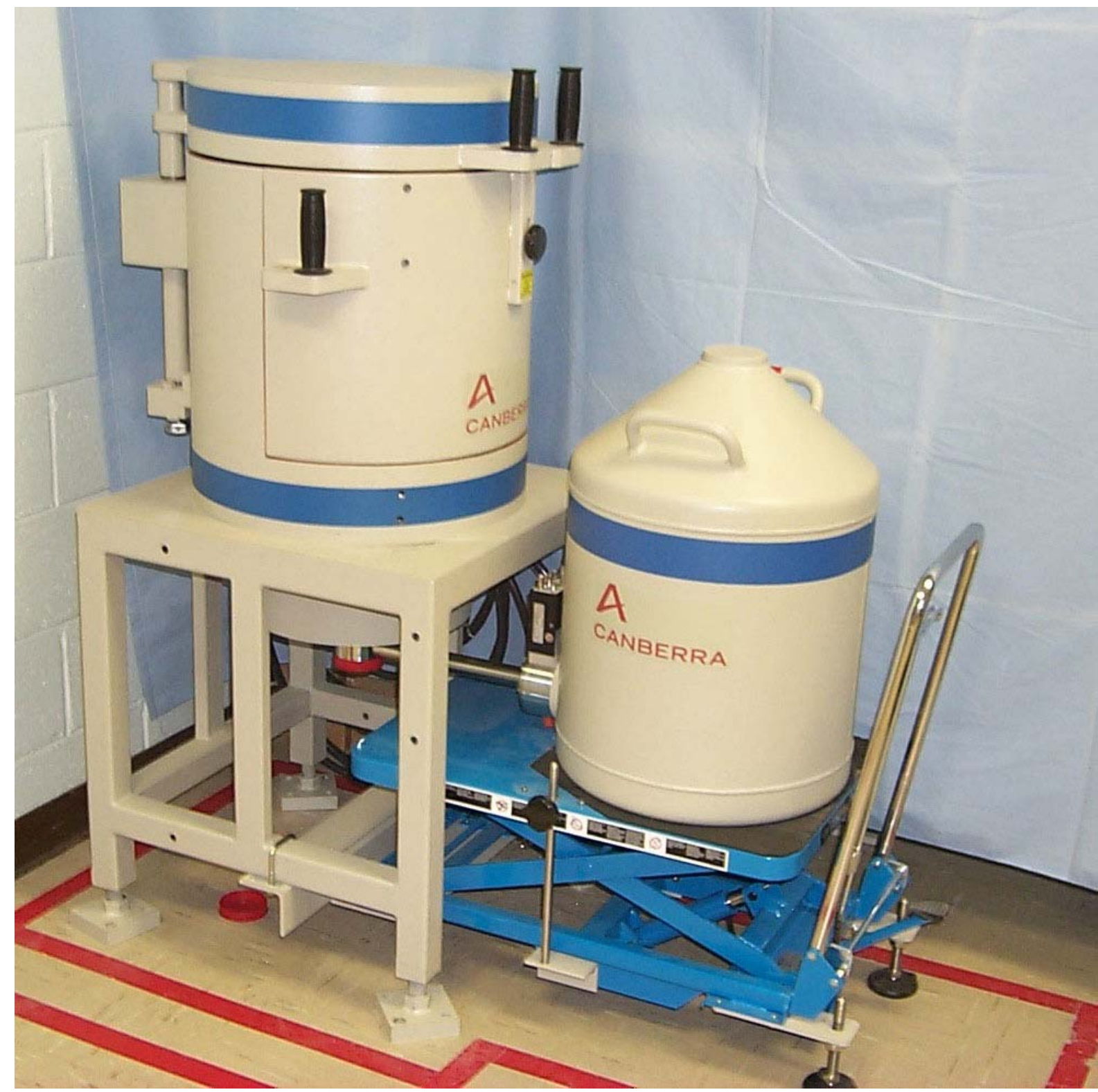

Figure A-1. Photograph of the assembled G3 spectrometer. The detector is raised into its measurement position. 


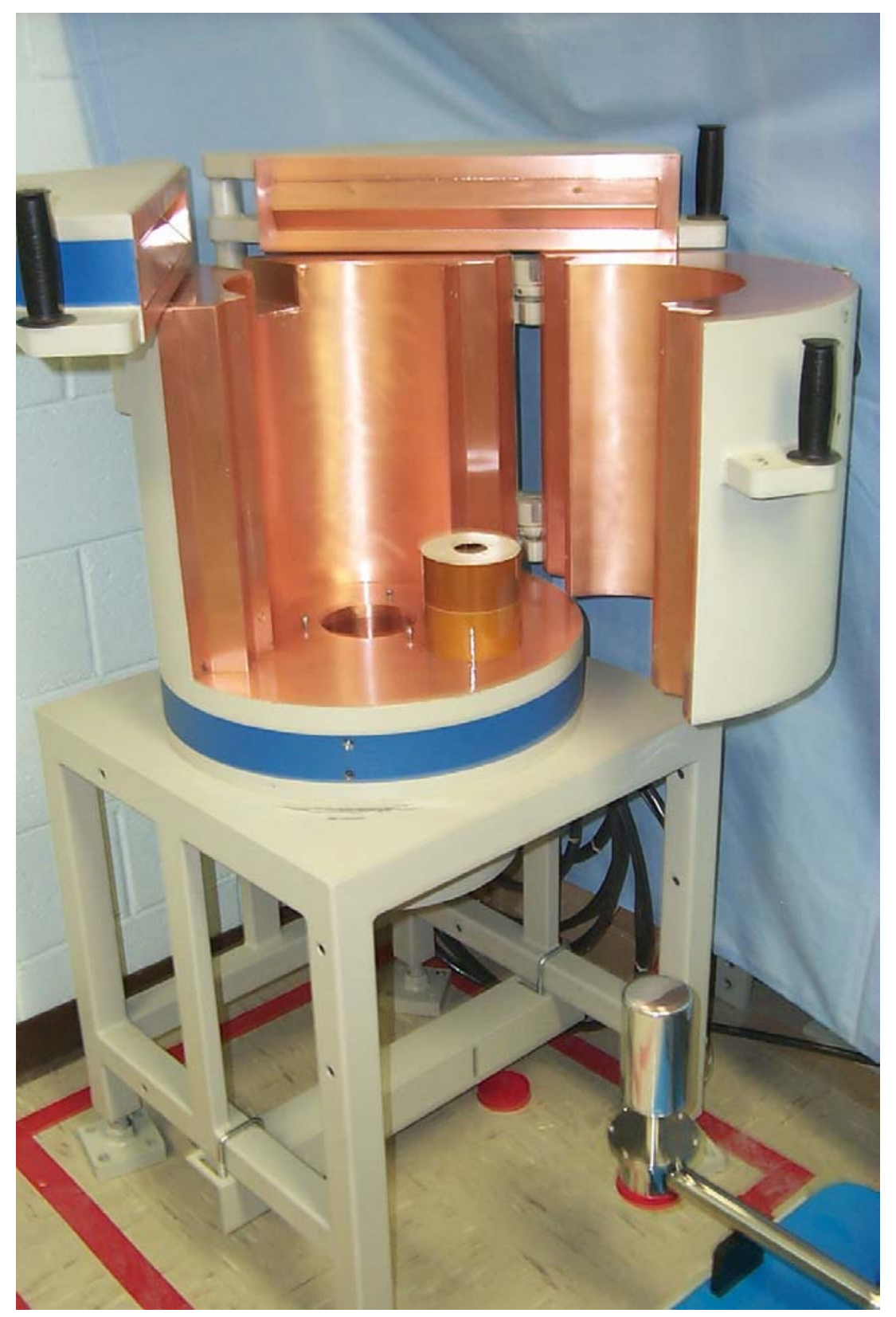

Figure A-2. G3 spectrometer with the front and top doors open, detector removed (detector head is visible extending in from bottom right), and the collimator removed from the bottom center of the shield floor. 


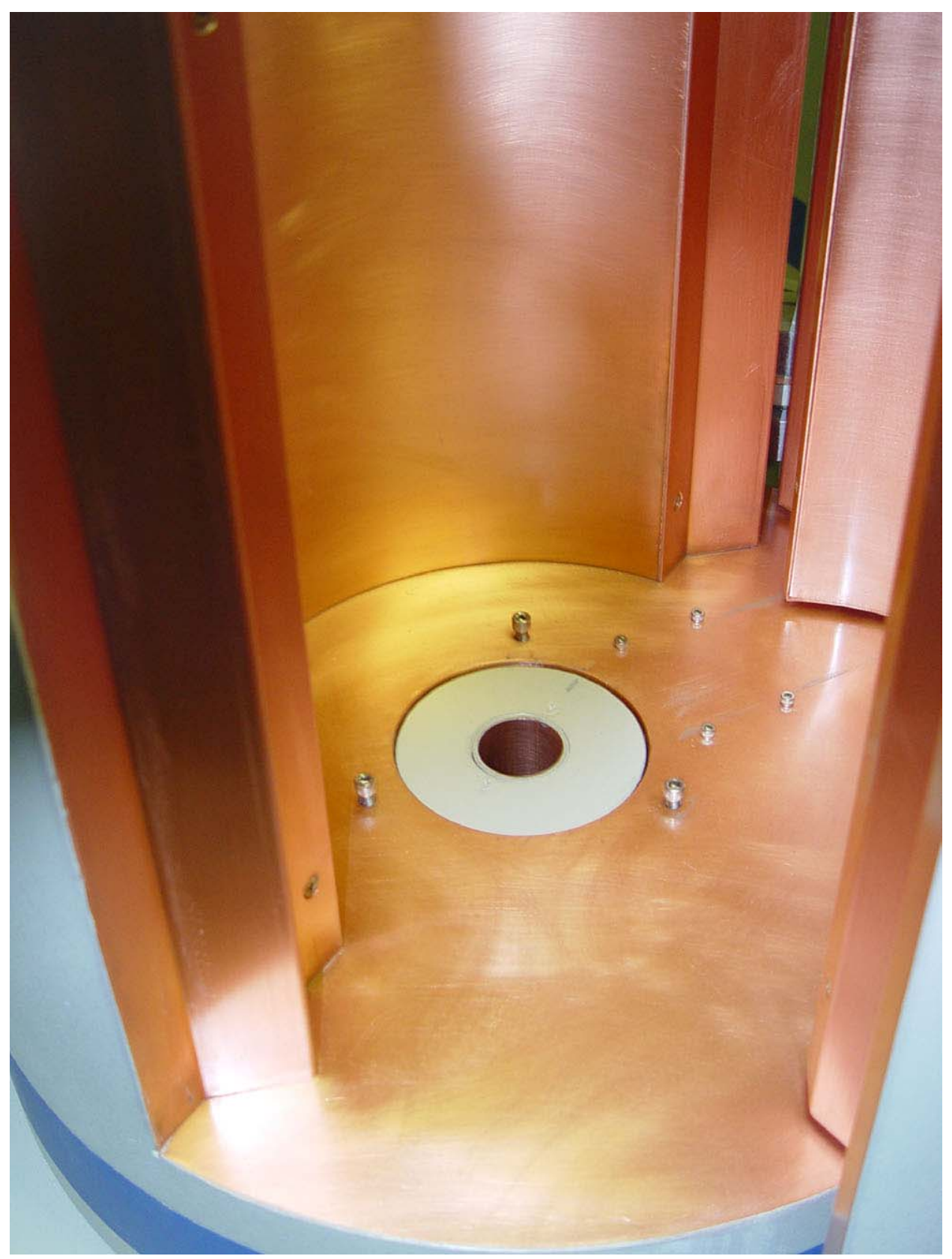

Figure A-3. Close up of the interior of the G4 spectrometer shield with the $32 \mathrm{~mm}$ collimator aperture in place. The detector face is positioned $127 \mathrm{~mm}$ below the interior face of the $114 \mathrm{~mm}$ thick collimator, thus is $13 \mathrm{~mm}(0.5 \mathrm{in})$ below the lower collimator face. 


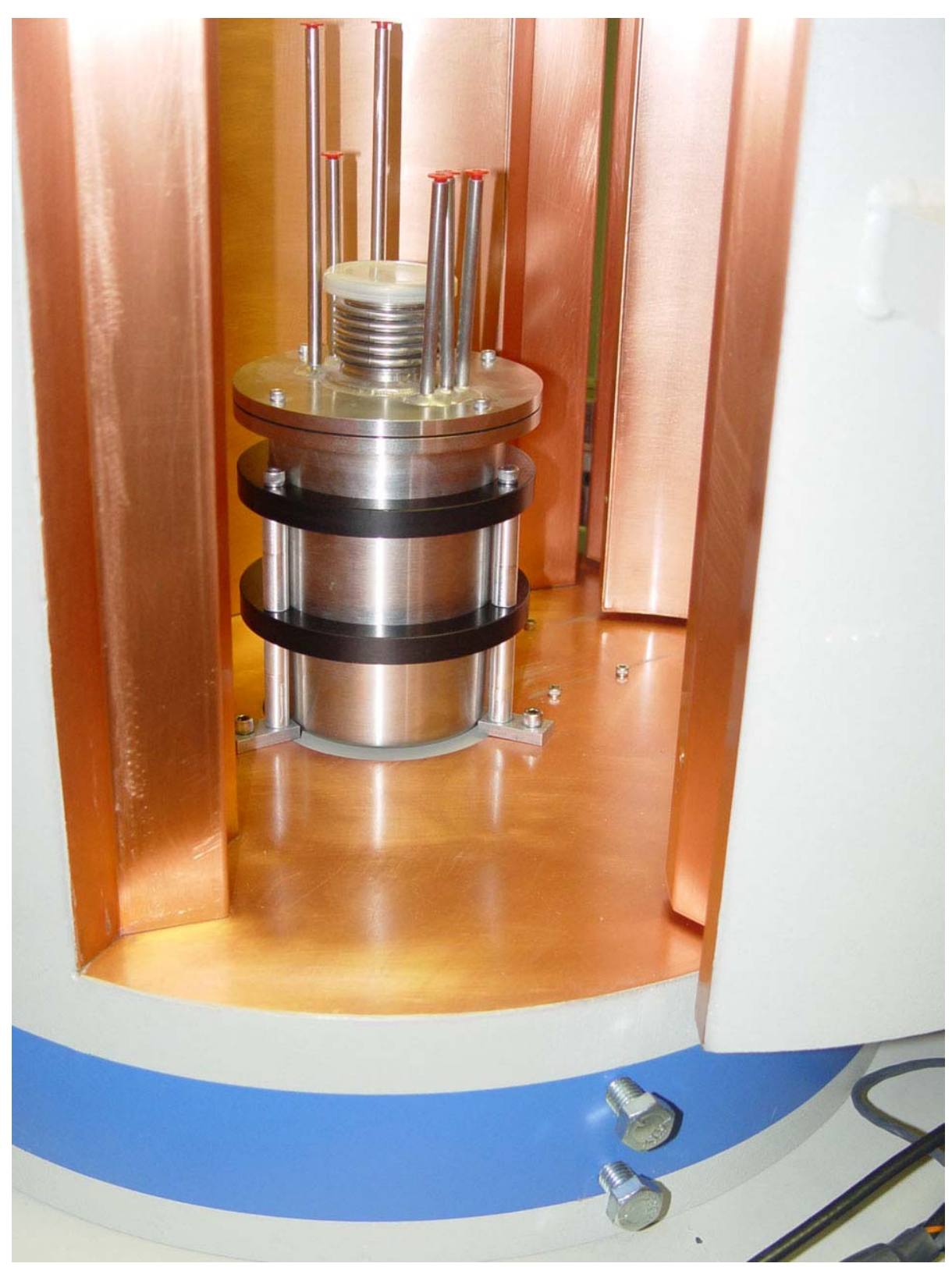

Figure A-4. The G4 spectrometer shield interior with a sample chamber similar (but not identical) to those to be used during AGR-1. This chamber has several flange penetrations that will not be in the AGR-1 design. The AGR-1 top flange will be penetrated only by a gas inlet tube and a gas outlet tube. 


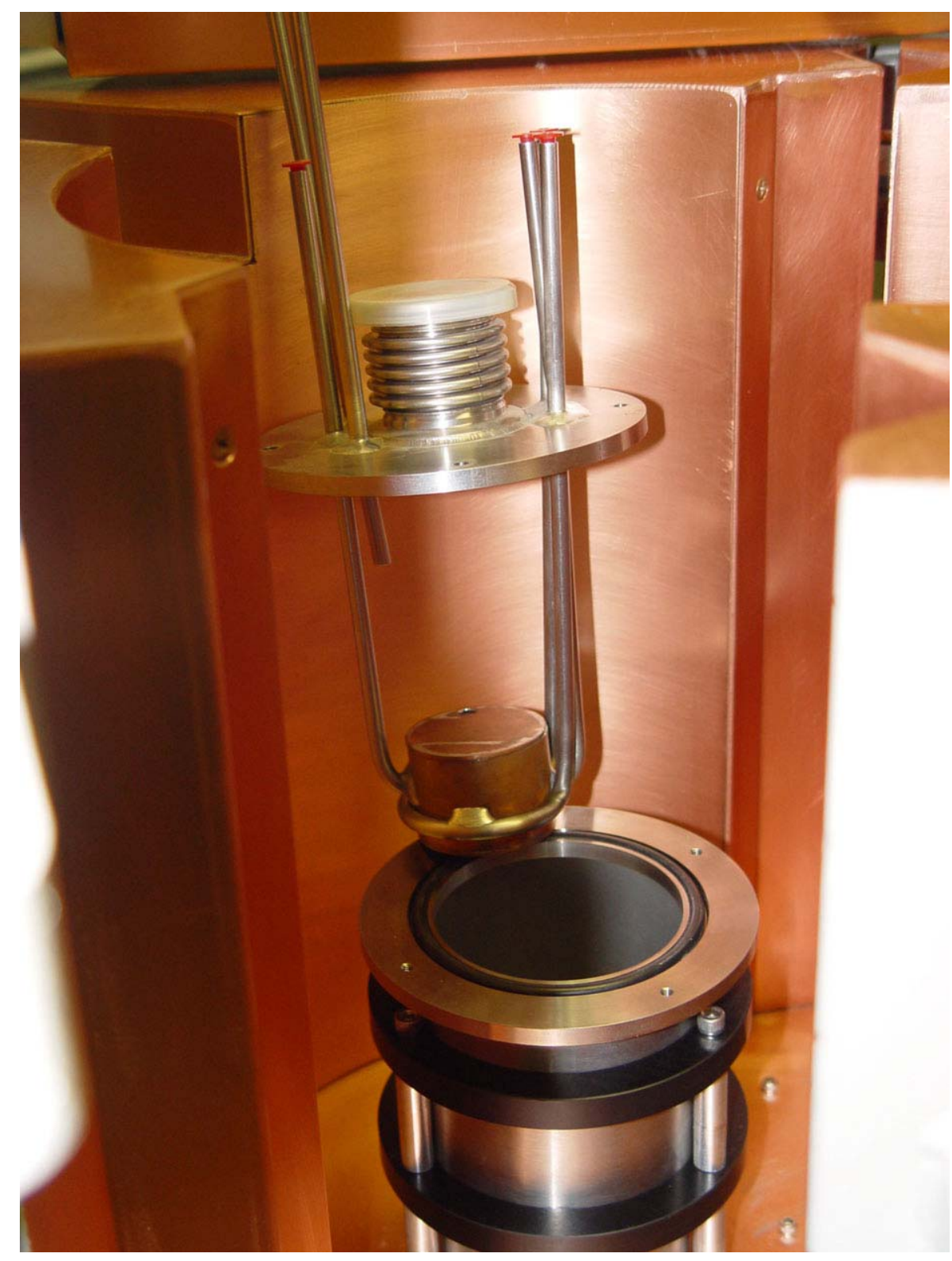

Figure A-5. Sample chamber interior view showing the flow-through $50 \mathrm{~cm}^{3}$ sample volume normally contained in the outer containment "beaker". 